\title{
Legislative Bargaining with Heterogeneous Disagreement Values: Theory and Experiments
}

\author{
Luis Miller*, Maria Montero ${ }^{\dagger}$ and Christoph Vanberg ${ }^{\ddagger}$ \\ This is the final version accepted by Games and Economic \\ Behavior, November 2017. The final publication is available at \\ Elsevier via https://doi.org/10.1016/j.geb.2017.11.003
}

\begin{abstract}
We study a legislative bargaining game in which failure to agree in a given round may result in a breakdown of negotiations. In that case, each player receives an exogenous 'disagreement value'. We characterize the set of stationary subgame perfect equilibria under all q-majority rules. Under unanimity rule, equilibrium payoffs are strictly increasing in disagreement values. Under all less-than-unanimity rules, expected payoffs are either decreasing or first increasing and then decreasing in disagreement values. We conduct experiments involving three players using majority and unanimity rule, finding qualitative support for several, but not all, of our main predictions. Having a high disagreement value is indeed an advantage under unanimity. Under majority, the player with the highest disagreement value is more likely to be excluded, but this does not consistently result in a lower average payoff.
\end{abstract}

Keywords: legislative bargaining; majority rule; unanimity rule; risk of breakdown; experiments.

JEL Classification: C78, C92, D71, D72.

${ }^{*}$ School of Economics, University of the Basque Country (Av. Lehendakari Aguirre 83, 48015 Bilbao, Spain; email: luismiguel.miller@ehu.es; Tel: +34 946013770).

${ }^{\dagger}$ School of Economics, University of Nottingham (University Park, Nottingham NG7 2RD, UK; e-mail: maria.montero@nottingham.ac.uk; Tel: +44 1159515468)

${ }^{\ddagger}$ Department of Economics, University of Heidelberg (Bergheimer Str. 58, 69115 Heidelberg, Germany; email: vanberg@uni-hd.de; Tel: +49 622154 2947) 


\section{Introduction}

Baron and Ferejohn (1989) proposed what appears to be the most influential formal model of legislative bargaining. ${ }^{1}$ In their model, a group of $n$ legislators is bargaining over the division of a dollar. Depending on the area of application, this dollar can be interpreted to represent a monetary amount (budget) or other items of value (allocation of ministries). In the basic (closed rule) version of the game, a randomly chosen player makes a proposal of how to divide the resource, and this proposal is voted on. If it passes, the game ends. If not, the game moves to a new round and the process is repeated.

An important feature of the Baron-Ferejohn game is that negotiations are assumed to continue until agreement is reached. In many real-world applications, however, failure to agree may lead, eventually, to a 'breakdown' of negotiations. In this case, the parties involved may shift to some other method of resolving the issue under consideration, or perhaps automatically revert to a 'status quo' situation. In what follows, we investigate what happens when the bargainers attach different values to the event of breakdown. More broadly, we will ask how the effects of heterogeneous 'disagreement values' depend on the decision rule being employed by the group.

As an example, consider debtor-creditor negotiations in a bankruptcy case. In the United States, the debtor may attempt to reorganize his business under Chapter 11. This requires that creditors agree to a reorganization 'plan'. Both the debtor and (under certain conditions) creditors may make repeated attempts at proposing and soliciting approval for such a plan. Should such attempts repeatedly fail, however, the court may terminate the proceedings and shift the case to Chapter 7, meaning that the business is liquidated and creditors receive payments according to the legal priority of their claims. ${ }^{2}$

In this and other examples, an important feature is that the payoffs that participants can anticipate in case of 'breakdown' are likely to differ. For example, if the business is liquidated under Chapter 7, some creditors can expect to receive larger payments than others, and those with low priority (as well as equity holders) may expect no payments at all. Another feature is that agreement may or may not be efficient, in the sense that the budget available in case of agreement may or may not exceed the sum of disagreement values. In the Chapter 11 example, agreement on a reorganization plan is efficient only if the expected sum of future payments to stakeholders (and perhaps further values they attach to the continued operation of the business), is larger than the anticipated liquidation value.

While bankruptcy negotiations are one example for the kind of problem we

\footnotetext{
${ }^{1}$ As of September 2017, Google Scholar counts over 2000 citations to the original paper.

${ }^{2} \mathrm{~A}$ similar procedure is used in Germany.
} 
wish to consider, it is important to emphasize that we do not intend to provide a model of the Chapter 11 decision procedures. ${ }^{3}$ Instead, we want to investigate, in a stylized setting, the effects of simple voting rules in a context where players attach different values to disagreement.

We investigate this issue by modifying the Baron-Ferejohn model in two ways. First, we assume that whenever a proposal fails, bargaining will continue with probability $\delta$, and otherwise "break down" (end without agreement). Second, we assume that in case of breakdown, each member receives an exogenously given non-negative payoff (disagreement value). ${ }^{4}$ Most importantly, these values are assumed to differ between members.

Among the questions of interest are: under what conditions will the outcome be efficient? If agreement is achieved, how is the resulting surplus shared among the participants? Most importantly, how do these outcomes depend on the decision rule being employed (e.g. unanimity vs. less-than unanimity rules)? ${ }^{5}$

Under unanimity rule, we find that efficiency is both necessary and sufficient for immediate agreement to occur. In expectation, the surplus resulting from an agreement is shared equally by all participants. That is, each player receives, in expectation, the sum of her disagreement value plus an equal share of the difference between the sum to be distributed and the sum of all disagreement values. Interestingly, this result is independent of the probability of breakdown.

Under all less-than-unanimity rules, results are quite different. In particular, efficiency is no longer necessary for immediate agreement to occur. In fact, it is possible for agreements to be reached which make even those that vote 'yes' worse off than they would have been in case of breakdown. ${ }^{6}$ When agreement is efficient, the resulting 'surplus' is not shared equally. Instead, the largest gains are captured by the players with the smallest disagreement values. The reason is that players with large disagreement values are often excluded from minimal

\footnotetext{
${ }^{3}$ Chapter 11 voting rules are far more complex than those considered here. In addition, the agreements reached are required to satisfy additional constraints which we will not incorporate into our model. Indeed, these constraints may help to prevent the kinds of outcomes that our model predicts would occur when simple (q-majority) voting rules are used.

${ }^{4}$ The disagreement value can be interpreted in several ways. One is that it represents the value that a player attaches to the status quo, which will prevail if agreement is not reached. Another is that it represents a reward directly associated with a failure to agree. For example, an individual may anticipate an extrinsic (social) or intrinsic (emotional) reward in case negotiations end with disagreement. In a government formation situation, a party's disagreement value may reflect its electoral prospects in the event a new election is called.

${ }^{5}$ Coming back to the bankruptcy example, it is interesting to note that Chapter 11 law requires only a (qualified) majority of creditors to accept a reorganization plan. In Germany, creditors are divided into 'groups' according to the type of claim they hold, and approval of the plan requires that all groups pass it using majority rule.

${ }^{6}$ An example is provided on p. 47 in the Appendix.
} 
winning coalitions. As a result, expected equilibrium payoffs are either decreasing in disagreement values (such that the player with the smallest disagreement value achieves the highest expected payoff), or first increasing and then decreasing (such that some other player, but never the one with the highest disagreement value, achieves the greatest payoff).

We test the predictions of our model within an experiment involving threeplayer games. In all games, the budget to be distributed in case of agreement is worth 100 'tokens' and the probability of breakdown is $\frac{1}{3}$. Each experimental session consists of several games involving three different sets of disagreement values, $(20,20,20),(0,20,40)$, and $(0,0,60)$. We compare behavior under unanimity and simple majority rule conditions.

The behavior we observe in the lab shows significant deviations from the theoretical predictions. While most proposals are immediately passed under majority rule, proposals under unanimity rule very often fail. The effect of the voting rule on the passage rate is larger than has been previously documented for 'standard' $\mathrm{BF}$ games. In addition, it is more pronounced when players have different disagreement values. Most proposers attempt to form minimal winning coalitions, but the proposed distribution between coalition members is more equal than the theory predicts. Proposals close to the theoretical prediction often fail in all treatments. Some of these rejections are clearly disadvantageous in terms of material payoffs, and this leads us to conclude that observed behavior cannot be explained by subgame perfect equilibrium, even if we drop the stationarity refinement and allow history-dependent strategies.

Despite the deviations from point predictions, our experimental results provide qualitative support for our hypotheses concerning the effect of disagreement values on payoffs. Consistent with our model, we find that the player with the largest disagreement value is offered more and achieves the largest payoff under unanimity rule. Also consistent with our model, that player is included in others' coalitions significantly less often under majority rule. Nonetheless, we do not find that this results in consistently lower average payoffs. A statistically significant disadvantage in terms of expected payoffs is found only in the $(0,0,60)$ condition. Overall, the results provide support for the theoretical prediction that having a high disagreement value is unambiguously beneficial under unanimity rule, but may be detrimental under majority rule.

\section{Related Literature}

Despite its simplicity, the BF game is rich in strategic possibilities and admits multiple subgame perfect equilibria. The theoretical literature has focused on symmetric stationary equilibria, which are (essentially) unique (Eraslan, 2002, and 
Norman, 2002). These equilibria are characterized by three empirically testable features. First, proposers form "minimal winning coalitions", allocating positive payoffs only to the number of players required to agree. Second, the distribution of payoffs within the coalition is unequal, favoring the proposer. Third, the first proposal passes immediately, so there is no delay. All three of these properties are independent of the decision rule being used (majority, qualified majority, or unanimity rule).

These (symmetric stationary subgame perfect) equilibrium properties of the BF model have been experimentally investigated in a number of papers (McKelvey, 1991, Fréchette et al, 2003, 2005a, 2005b, Diermeier and Morton 2005, Agranov and Tergiman, 2014, and Bradfield and Kagel, 2015). All of these studies investigated the simple majority rule version of the game. The central findings include: (1) Subjects do indeed form minimal winning coalitions, allocating positive shares to a bare minimum of players. (2) The distribution of shares within the majority coalition is generally more equal than theory predicts ${ }^{7}$, and (3) The vast majority of games end in immediate agreement to the first proposal made.

Building on this literature, Miller and Vanberg (2013, and 2015) investigated the effects of different decision rules (majority and unanimity rule) within experimental BF games. Inspired by Buchanan and Tullock (1962), their main hypotheses were (a) that unanimity rule would be associated with greater costly delay in reaching agreement, and (b) that this effect would be driven in part by "tougher" bargaining at the individual level. The main finding from these studies was that unanimity rule is indeed associated with significantly greater delay. Both studies also find some support for the notion that individuals adopt a 'tougher' stance under unanimity rule, more often voting 'no' on a given proposal and making less generous offers when proposing. Most importantly, and consistent with prior experiments, both studies find relatively little delay overall, as well as a tendency to agree on symmetric distributions - most commonly an equal split within a minimal winning coalition. ${ }^{8}$

The effect of heterogeneous disagreement values in the event of a breakdown of negotiations has been studied theoretically for the case of two players (see Binmore et al., 1986); these results can be easily extended to $n$ players and unanimity rule. ${ }^{9}$

\footnotetext{
${ }^{7}$ These relatively equal allocations cannot be explained by inequity aversion (Montero, 2007) but are consistent with a model of noisy best response (Nunnari and Zapal, 2016). Agranov and Tergiman (2014) find that communication brings outcomes closer to the theoretical prediction.

${ }^{8}$ The effect of allowing communication depends on the voting rule (Agranov and Tergiman, 2016). While communication significantly increases proposer power under majority rule due to competition between potential coalition partners, it has the opposite effect under unanimity rule: proposer shares decrease and allocations become more equal. Communication also eliminates costly delays under unanimity rule.

${ }^{9}$ Binmore et al. (1986) use the alternating-offer procedure of Rubinstein (1982) rather than
} 
To the best of our knowledge, the case of general $q$-majority rules has not been studied. The closest paper we are aware of is Banks and Duggan (2006). In their model, players receive a flow payoff every bargaining period; for every period in which agreement has not been reached, they receive a status quo payoff. They prove existence of SSPE for very general policy spaces and voting rules. In one of the special cases they consider (model 5 ), the policy space is the unit simplex and the decision rule is simple majority; this is similar to our model with the additional restriction $q=\frac{n+1}{2}$ ( $n$ odd). They discuss only equilibria in which all players have the same continuation value, noting that the equilibrium may or may not be of this type depending on the parameters. We provide a full characterization for arbitrary values of the parameters and of $q .^{10}$

A different way to model a situation where some players have less to lose than others if agreement is delayed is to introduce heterogeneous discount factors. Intuitively, a player with a greater discount factor has less to lose from delay since the value of the pie is less heavily discounted; similarly, a player with a greater disagreement value has less to lose from delay since he receives a greater payoff in the event of a breakdown of negotiations. An important difference is that in the case of heterogeneous discount factors, immediate agreement is always efficient, and no player can be harmed by an agreement compared to the situation of perpetual disagreement. The two-player game with asymmetric discount factors (and unanimity) was solved by Rubinstein (1982) for the case of alternating offers; the random proposers case is very similar (Binmore, 1987). The BaronFerejohn model with possibly asymmetric discount factors and general $q$-voting rules has been studied in Eraslan (2002) and Kalandrakis (2015); both papers establish uniqueness of equilibrium payoffs using different methods. Continuation values are nondecreasing in discount factors (Eraslan, 2002); similarly, we find that continuation values are nondecreasing in disagreement values. For the case of unanimity rule, expected equilibrium payoffs are increasing in the discount factors (Eraslan, 2002). However, under majority rule, expected equilibrium payoffs are

the random proposer procedure of Binmore (1987), Baron and Ferejohn (1989) and Okada (1996). This makes little difference to the results under unanimity rule, namely that a player benefits from having a higher disagreement value, and that disagreement values remain relevant even as the breakdown probability goes to zero.

${ }^{10}$ Harstad (2010) analyzes a different model with a continuum of districts where voters in each district choose a representative, representatives form a majority coalition, and the majority coalition then decides on the distribution of tax revenue and the possible implementation of a public project. The representative may be a conservative (a player who is biased in favor of the status quo, similar to a player with a high disagreement value in our model) or a progressive (a player who is biased in favor of undertaking the public project). A progressive is more likely to be included in the majority coalition, while a conservative will get a better deal conditional on being included. Voters in a district tend to elect a conservative if the majority requirement is large (not necessarily unanimity), and a progressive if the majority requirement is small. 
decreasing in discount factors provided that the discount factors are sufficiently high (Kawamori, 2005); this result is analogous to our result that, when the continuation probability is sufficiently close to 1 , expected equilibrium payoffs are decreasing in disagreement values (Appendix A.4). We analyze expected equilibrium payoffs more fully in our setting, since we do not restrict ourselves to the limit case in which the continuation probability is sufficiently close to 1 , and we are able to show that there are only two possibilities for expected equilibrium payoffs: they are either decreasing in disagreement values, or they are first increasing and then decreasing in disagreement values.

A small number of experimental studies have introduced asymmetries into the BF framework. Diermeier and Morton (2005) and Fréchette et al. (2005a) consider the case in which some players are more likely to be selected as proposers. Other papers consider voting rules in which some players are favored, either by being given veto power (Kagel et al., 2010) or by having more votes than other players (Fréchette et al., 2005c, and Drouvelis et al., 2010). In some cases, having more votes than other players does not theoretically confer any objective advantage, and subject behavior broadly confirms this (Diermeier and Morton, 2005, Fréchette et al., 2005b, and Drouvelis et al., 2010). Diermeier and Gailmard (2006) study a 1-round version of the game in which failure to agree results in exogenously given payoffs that differ between players. This is a particular case of our model presented below with $\delta=0$ (i.e. certain breakdown). Some of their findings are similar to ours, in particular, the player with the highest disagreement value is often excluded. They also report that proposers demand more when their own disagreement value is greater, contrary to equilibrium predictions, and responders are more accepting of offers when the proposer's disagreement value is higher.

The Baron-Ferejohn model has been extended in many other directions. Examples include the allocation of resources between public and private goods (Volden and Wiseman (2007, 2008); tested experimentally by Fréchette et al. (2012) and Christiansen (2015)), the tradeoff between decisions on ideological and redistributive dimensions (Jackson and Moselle (2002); tested experimentally by Christiansen et al. (2013)), combining bargaining and joint production (Baranski (2016)), and repeated negotiations such that, if agreement fails on a particular period, last period's agreement prevails for the current period (Kalandrakis (2004), Battaglini and Palfrey (2012)). Battaglini et al. (2012) compare voting rules in a dynamic setting where resources can be invested in a durable public good or in targeted transfers. The model predicts that more investment goes into the public good as the majority required to pass a proposal increases, and this prediction is supported by the experimental results. 


\section{Model}

Let $N=\{1,2, \ldots, n\}$ be the set of players and $q$ be the number of votes needed to pass a proposal, where $1<q \leq n$. Within each round, Nature selects a proposer randomly, with each player having a $\frac{1}{n}$ probability of being selected. The proposer can propose any vector $x=\left(x_{1}, \ldots, x_{n}\right)$, provided that $x_{i} \geq 0$ for all $i$ and $\sum_{i \in N} x_{i} \leq 1$. All players vote on the proposal. If there are at least $q$ votes in favor, the proposal passes. Otherwise, there is a probability $0 \leq \delta<1$ that bargaining moves to the next round, and a probability $(1-\delta)$ that breakdown occurs, in which case payoffs are given by a vector $r=\left(r_{1}, \ldots, r_{n}\right)$ of disagreement values. Players are labeled so that $0 \leq r_{1} \leq r_{2} \leq \ldots \leq r_{n}$. The game continues until either a proposal is accepted, or breakdown occurs. Note that under all decision rules, agreement is efficient if and only if $\sum_{i \in N} r_{i} \leq 1$.

\subsection{Equilibrium concept}

A strategy in this game specifies a) what proposal a player makes when recognized as proposer, and b) how he would vote on any proposal made by other players. In principle, these actions could depend upon the history of play. For example, the proposal that a player makes in a given round may depend on prior proposals or voting decisions. Following the prior literature on legislative bargaining, we will exclude such behavior and focus on equilibria in which players use stationary strategies. Such strategies require that players make the same (possibly random) proposal in each round, and vote the same way on others' proposals. A subgame perfect equilibrium in which players use stationary strategies is called a stationary subgame perfect equilibrium (SSPE). Additionally, we assume that all players vote as if they are pivotal.

Following common practice, we will refer to a player's expected utility given that a proposal has (just) been rejected as the player's continuation value. For any profile of stationary strategies (equilibrium or not), there is an associated vector of continuation values (the same in all rounds) and an associated vector of expected payoffs computed at the beginning of the game, before Nature selects a proposer. Given a profile of stationary strategies $\sigma$, we will denote player $i$ 's continuation value as $z_{i}(\sigma)$ and player $i$ 's expected payoff as $y_{i}(\sigma)$; we will drop $\sigma$ from the notation if no confusion arises.

In a SSPE, continuation values act as prices: it is optimal for player $i$ to vote in favor of any proposal with $x_{i} \geq z_{i}$, and to vote against otherwise. As a proposer, player $i$ looks for the $q-1$ players with the lowest $z_{j}$ and sets $x_{j}=z_{j}$ for these players, keeping the remainder. If the remainder is below $z_{i}$, it is better for player 
$i$ to make a proposal that will be rejected and get $z_{i}{ }^{11}$ We can then distinguish between equilibria with no delay or immediate agreement (in which all players prefer to make a proposal that will be accepted) and equilibria with delay (in which at least one player prefers to make a proposal that will be rejected, and hence disagreement occurs with positive probability).

\subsection{Example}

Before presenting a general analysis, we will illustrate our main results using a simple example. Assume that the group consists of $n=3$ players, with disagreement values $r=\left(0,0, \frac{1}{2}\right)$. Player 3 , having the largest disagreement value, can be thought of as 'tougher' than the other players. Let the continuation probability be given by $\delta=2 / 3$. Thus, in case a proposal fails, the game will end without agreement with probability $\frac{1}{3}$.

Suppose first that the group makes decisions using unanimity rule. Then, there exists a stationary subgame perfect equilibrium in which the continuation values are $z=\left(\frac{2}{18}, \frac{2}{18}, \frac{11}{18}\right)$. The strategies are as follows. If, say, player 1 is selected as a proposer, he offers $\frac{2}{18}$ to player 2 and $\frac{11}{18}$ to player 3 , keeping $1-\frac{2}{18}-\frac{11}{18}=\frac{5}{18}$. Similarly, player 3 would offer $\frac{2}{18}$ to each of the other two players and keep $\frac{14}{18}$. Given these strategies, expected payoffs are $y_{1}=y_{2}=\frac{1}{3} \frac{5}{18}+\frac{2}{3} \frac{2}{18}=\frac{3}{18}$ and $y_{3}=$ $\frac{1}{3} \frac{14}{18}+\frac{2}{3} \frac{11}{18}=\frac{12}{18}$. We can check that the $z$ values we have provided are indeed the continuation values for the players. Given the strategies, $z_{1}=z_{2}=\frac{2}{3} \frac{3}{18}+\frac{1}{3} 0=\frac{2}{18}$ and $z_{3}=\frac{2}{3} \frac{12}{18}+\frac{1}{3} \frac{1}{2}=\frac{11}{18}$.

Under unanimity rule, we see that the players' expected payoffs are increasing in disagreement values. Player 3, being the 'toughest' player, is more expensive to 'buy' into a coalition. Since all players have to be 'bought', he is paid more for his vote than the other two players. Under unanimity rule, it is good to be the 'toughest' player (to have a large disagreement value).

Next, suppose that the group decides using majority rule. In this case, there exists a stationary subgame perfect equilibrium in which the continuation values are $z=\left(\frac{1}{4}, \frac{1}{4}, \frac{1}{3}\right)$. As in the case of unanimity rule, these continuation values are increasing in the disagreement values. Again, player 3's vote is 'more expensive' than that of the other two players. However, in the case of majority rule, this causes the other players to exclude player 3 from the coalition when they propose. If player 1 or 2 proposes, 3 is excluded and the other player is offered $\frac{1}{4}$. If player 3 proposes, he offers one of the other players $\frac{1}{4}$ (with equal probability). Expected payoffs are then $y_{1}=y_{2}=\frac{1}{3}\left[1-\frac{1}{4}\right]+\frac{1}{3} \frac{1}{4}+\frac{1}{3} \frac{1}{2} \frac{1}{4}=\frac{3}{8} ; y_{3}=\frac{1}{3}\left[1-\frac{1}{4}\right]=\frac{1}{4}$. Again, it can

\footnotetext{
${ }^{11}$ In case of indifference, we assume that players break ties in favor of agreement, that is, responders vote in favor of proposals with $x_{i}=z_{i}$ and proposers offer $q-1$ players their continuation value if the remaining payoff $x_{i}=z_{i}$. This assumption simplifies the analysis and makes little difference to equilibria; see Appendix A.1.
} 
be easily checked that these expected payoffs are consistent with the conjectured continuation values.

Under majority rule, the players' expected payoffs are not increasing in their disagreement values. Player 3's continuation value is larger, making his vote more expensive. This is why he is excluded whenever others propose. His expected payoff under majority rule is smaller than that of the other players. Under majority rule, being 'tough' (having a large disagreement value) can be a disadvantage.

To summarize, the example demonstrates a set of patterns that turn out to be more general. Under all decision rules, continuation values $z$ are (weakly) increasing in disagreement values. Under unanimity rule, the same is true for expected payoffs $y$. Under all less-than-unanimity rules, by contrast, expected payoffs are either decreasing or non-monotone in disagreement values, and the player with the largest disagreement value never achieves the greatest payoff. ${ }^{12}$ The following subsection establishes that all equilibria without delay satisfy these properties. Equilibria with delay are discussed in Appendix A.5.

\subsection{Equilibria with no delay}

Recall that a no-delay equilibrium is one in which all players make proposals that pass. In order to characterize the properties of such equilibria, as well as the conditions under which they occur, it will be useful to consider how the payoffs $y_{i}$, continuation values $z_{i}$ and disagreement values $r_{i}$ are related to one another.

When a proposal passes, we refer to the players who vote in favor as the coalition that forms, and to players in the coalition other than the proposer as the coalition partners. In any no-delay equilibrium, coalition partners receive $z_{i}$, and the proposer receives $1-\sum_{j \in T \backslash\{i\}} z_{j}$, where $T$ is (one of) the 'cheapest' coalition(s) that includes $i .^{13}$ All other players receive 0 .

As we show in Appendix A.2, equilibrium continuation values are weakly increasing in the disagreement values, i.e.,

$$
z_{1} \leq z_{2} \leq \ldots \leq z_{n}
$$

It follows that (one of) the cheapest coalition(s) a player can buy consist of himself plus the 'first' $(q-1)$ other players (those with the smallest continuation values $z_{j}$ ). Let $Z_{q}=\sum_{j=1}^{q} z_{q}$. This would be the minimum 'price' to pay for the first $q$ votes. However, player $i$ must buy only $(q-1)$ votes. If his own continuation value is no larger than $z_{q}$, he must pay $Z_{q}-z_{i}$ for his cheapest coalition(s) (he

\footnotetext{
${ }^{12}$ Another general pattern is that a player's payoff is larger if he proposes than when he is a coalition partner.

${ }^{13}$ If the cheapest coalition is not unique, player $i$ can mix. By definition, the total 'price' of all cheapest coalitions is the same.
} 
need not pay himself). If his own continuation value is strictly larger than $z_{q}$, he must pay $Z_{q}-z_{q}$ (he need not buy player $q$ ). It follows that player $i$ 's payoff as a proposer is $\left(1-Z_{q}+\min \left\{z_{i}, z_{q}\right\}\right)$.

We denote the ex ante probability of player $i$ being in the coalition that forms by $\mu_{i}$. Naturally, player $i$ will be in the coalition whenever he is the proposer; this occurs with probability $\frac{1}{n}$. Then the equilibrium probability of $i$ being a coalition partner is $\mu_{i}-\frac{1}{n}$. As a coalition partner, player $i$ 's payoff is given by $z_{i}$.

From what we have said so far, it follows that the expected equilibrium payoff for player $i$, denoted $y_{i}$, is related to the continuation values and inclusion probabilities as follows.

$$
y_{i}=\frac{1}{n}\left(1-Z_{q}+\min \left\{z_{i}, z_{q}\right\}\right)+\left(\mu_{i}-\frac{1}{n}\right) z_{i}
$$

In case a proposal fails, bargaining continues with probability $\delta$, in which case players' expected payoffs are again given by $y_{i}$. Bargaining will break down with probability $1-\delta$, in which case players receive their disagreement value $r_{i}$. Therefore, player $i$ 's continuation value is given by

$$
z_{i}=\delta y_{i}+(1-\delta) r_{i}
$$

Combining these equations yields $n$ equations relating the vector of continuation values $z=\left(z_{1}, \ldots, z_{n}\right)$ to the vector of inclusion probabilities $\mu=\left(\mu_{1}, \ldots, \mu_{n}\right)$. Specifically,

$$
z_{i}=\left\{\begin{array}{ll}
\frac{1}{1-\delta \mu_{i}}\left((1-\delta) r_{i}+\frac{\delta}{n}\left(1-Z_{q}\right)\right) & z_{i} \leq z_{q} \\
\frac{1}{1-\delta\left(\mu_{i}-\frac{1}{n}\right)}\left((1-\delta) r_{i}+\frac{\delta}{n}\left(1-Z_{q}+z_{q}\right)\right) & z_{i}>z_{q}
\end{array} \quad i=1, \ldots, n\right.
$$

In equilibrium, the inclusion probabilities $\mu$ must reflect the fact that proposers will buy the cheapest available coalition given $z$. An equilibrium can therefore be constructed as follows. (Details are presented in Appendix A.3.)

Begin by conjecturing that $L \in\{0,1, \ldots, q-1\}$ players are strictly cheaper than player $q$. For these players, we must have $\mu_{i}=1$ as they are included in all coalitions. Then for these players

$$
z_{i}=\frac{1}{1-\delta}\left((1-\delta) r_{i}+\frac{\delta}{n}\left(1-Z_{q}\right)\right) \quad i=1, \ldots, L
$$

Also assume that $H \in\{0,1, \ldots, n-q\}$ players are strictly more expensive than player $q$. For these players, we must have $\mu_{i}=\frac{1}{n}$ as they are included only in their own coalitions. Therefore

$$
z_{i}=(1-\delta) r_{i}+\frac{\delta}{n}\left(1-Z_{q}+z_{q}\right) \quad i=n-H+1, \ldots, n
$$


The remaining $M=n-L-H \geq 1$ players are exactly as expensive as player $q$ and so we have

$$
z_{q}=\frac{1}{\left(1-\delta \mu_{i}\right)}\left((1-\delta) r_{i}+\frac{\delta}{n}\left(1-Z_{q}\right)\right) \quad i=L+1, \ldots, n-H
$$

Although the individual inclusion probabilities for these players are not immediately determined, it's clear that the average inclusion probability for these players must be exactly large enough to achieve an expected coalition size of $q$. Specifically, it must equal $\bar{\mu}_{M}=\frac{q-L-\frac{H}{n}}{n-H-L}$. Therefore the corresponding equations can be combined to yield

$$
z_{q}=\frac{1}{\left(1-\delta \bar{\mu}_{M}\right)}\left((1-\delta) \bar{r}_{M}+\frac{\delta}{n}\left(1-Z_{q}\right)\right)
$$

where $\bar{r}_{M}=\frac{1}{n-H-L} \sum_{i=L+1}^{n-H} r_{i}$ is the average disagreement value among those players for whom $z_{i}=z_{q}$.

Suppose for example that $M=n$. Then, $\bar{\mu}_{M}=\frac{q}{n}$ and $Z_{q}=q z_{q}$. The last equation then reduces to $z_{q}=(1-\delta) \bar{r}_{N}+\frac{\delta}{n}$. This makes sense, since with immediate agreement, $\sum_{i \in N} z_{i}=\delta+(1-\delta) \sum_{i \in N} r_{i}$. If $M=n$, all players have the same continuation value, which must then equal the previous expression divided by $n .{ }^{14}$

In similar fashion, the equations derived above can be solved explicitly for any conjecture regarding the numbers $L, M$, and $H$. Subsequently, the resulting vector of continuation values can be inspected to verify that the first $L$ players are strictly cheaper than player $q$, etc. If so, the combination of continuation values and implied inclusion probabilities constitute a SSPE of the model. We show in the Appendix that each conjecture regarding $L, M$, and $H$ indeed uniquely determines the continuation values and inclusion probabilities (proposition 3). Furthermore, there is only one combination of $L, M$, and $H$ that leads to an equilibrium (proposition 5). Hence, all no-delay SSPE have the same payoffs. Based on our analysis, we are able to construct equilibria for all constellations of the parameters $r_{i}$ and $\delta$. In what follows, we concentrate on stating our main results.

\subsection{Unanimity rule $(q=n)$}

Under unanimity rule, immediate agreement occurs in equilibrium if and only if it is efficient, and both continuation values and expected payoffs are increasing in disagreement values.

\footnotetext{
${ }^{14}$ As mentioned in Section 2, this is the special case of our model considered by Banks and Duggan (2006). We provide a more complete characterization.
} 
Proposition 1. For $q=n$, immediate agreement occurs if and only if $\sum_{i \in N} r_{i} \leq$ 1. In this case, the SSPE is unique. ${ }^{15}$ Expected equilibrium payoffs are given by

$$
y_{i}=\frac{1}{n}\left(1-\sum_{i \in N} r_{i}\right)+r_{i},
$$

and continuation values are given by

$$
z_{i}=\frac{\delta}{n}\left(1-\sum_{i \in N} r_{i}\right)+r_{i} .
$$

If $\sum_{i \in N} r_{i}>1$, disagreement occurs with probability 1 and $z_{i}=y_{i}=r_{i}$.

Proof. See Appendix A.6.

Note that the expected payoffs are independent of $\delta$, such that the profile of disagreement payoffs $\left(r_{i}\right)_{i \in N}$ remains relevant even if the continuation probability becomes arbitrarily close to 1 . For all $\delta$, players effectively share the surplus from agreement equally. This result is analogous to the results for two-player bargaining with breakdown probability. Most importantly, note that expected equilibrium payoffs are increasing in disagreement values.

Although expected payoffs do not depend of $\delta$, the outcome of the game, conditional on the identity of the proposer, does. This can be seen by comparing the expressions for $y_{i}$ and $z_{i}$. Since $z_{i}<y_{i}$, it follows that the payoff conditional on being a responder is lower than that conditional on being proposer. This difference, which indicates a proposer advantage, is decreasing in $\delta$ and vanishes in the limit when $\delta$ approaches 1 .

\subsection{Less-than-unanimity rules $(q<n)$}

For less-than-unanimity rules, we can establish a sufficient condition for immediate agreement. Under unanimity rule, immediate agreement occurred if it was potentially Pareto improving. Under majority rule, an analogous condition holds that takes into account that only $q$ players need to agree in order for a proposal to be implemented: if $\sum_{i=1}^{q-1} r_{i}+r_{n}<1$, each player can find a coalition of $q$ players to which they belong for which $\sum_{i \in S} r_{j}<1$, hence each proposer can find a coalition for which agreement is potentially Pareto improving, and all equilibria involve immediate agreement in this case.

We also show that, even though SSPE with immediate agreement may differ in the strategies that are played, they all lead to the same expected payoffs and continuation values for the players.

\footnotetext{
${ }^{15}$ For the case $\sum_{i \in N} r_{i}=1$, the tie-breaking rule is used to select a unique equilibrium.
} 
Finally, we establish a crucial difference between unanimity and less-thanunanimity decision rules: even though continuation values are ranked in the same way as disagreement values, expected equilibrium payoffs are not. Expected equilibrium payoffs follow one of two basic patterns: they are either decreasing, or first increasing and then decreasing in disagreement values (in both cases, there may be an additional flat region after the decreasing part). The player with the greatest disagreement value never gets the highest expected payoff.

Proposition 2. Let $q<n$.

(i). If $\sum_{i=1}^{q-1} r_{i}+r_{n}<1$, all SSPE exhibit immediate agreement.

(ii). All SSPE with immediate agreement have the same continuation values and expected equilibrium payoffs.

(iii). Continuation values are weakly increasing in the r-values in any SSPE.

(iv). Inclusion probabilities are weakly decreasing in the $r$-values in any SSPE.

(v). In an SSPE with immediate agreement, the player with the greatest disagreement value never gets the highest expected payoff.

Proof. See Appendix A.6.

It follows from Propositions 1 and 2 that efficiency of agreement (i.e., $\sum_{j \in N} r_{j}<$ 1 ) is a sufficient condition for immediate agreement under all decision rules. However, under all less-than-unanimity rules, agreement may occur even when it is inefficient. In fact, it is possible that an agreement is reached which makes all players (including those that vote in favor) worse off than they would be in case of breakdown. (See p. 47 in the Appendix for an example.)

\section{Experimental Design and Hypotheses}

\subsection{Experimental Design}

We conducted several games involving three players with a divisible amount of 100 'tokens'. The continuation probability was $\delta=\frac{2}{3} \cdot{ }^{16}$ Sessions were divided into three blocks of ten games, corresponding to three different sets of disagreement values: $r=(20,20,20), r=(0,20,40)$, and $r=(0,0,60)$. (Instructions referred to "default tokens" which the subject would receive if the group failed to agree.) Thus, we are focusing on situations where agreement is efficient. ${ }^{17}$ In each of

\footnotetext{
${ }^{16}$ This was implemented using a virtual die roll, with the game continuing until the number rolled exceeded four.

${ }^{17}$ Our working paper reports on a previous experiment in which $r$ values were randomly assigned. This resulted in a larger number of distinct situations, including some where agreement was inefficient. A disadvantage of that design was that the constellation of $r$ values varied so much that subjects had little opportunity to learn. At the suggestion of a referee, we therefore
} 
the situations implemented, the surplus resulting from agreement is identical (40 tokens). Furthermore, the situations are clearly ranked in terms of the inequality of disagreement values, from fully equal to highly unequal. To facilitate learning, each subject's individual $r_{i}$ value was fixed over all games within a block. ${ }^{18}$

Within each game, the sequence of events was as follows. First, each subject was randomly assigned a letter i.d. ('A', 'B', 'C') which remained fixed throughout the game. Each subject was informed about the disagreement values for all members of the group. At the beginning of a given round of the game, one subject was randomly chosen to make a proposal. All subjects were immediately informed of the proposer's i.d., and the chosen subject was prompted to enter a proposal, consisting of three positive integers which sum to at most 100 tokens. After the proposer clicked 'ok', this proposal was displayed to all members of the group, who were then prompted to vote either 'yes' or 'no'. Following this, detailed results of the vote were displayed to all subjects. This included the proposal made, the i.d. of the proposer, and the individual votes cast by each player ('A','B', 'C'). If the proposal failed, the results screen also informed subjects of the outcome of a die roll to determine whether the game would continue. If so, a new round began. In games that continued beyond round 1, a detailed history table showed the proposer i.d., the proposal made, as well as the individual votes, for all prior rounds.

We conducted ten sessions, five using majority and five unanimity rule. Each session involved 24 subjects divided into two matching groups of size 12 . Thus, we have a total of 20 matching groups, 10 under each decision rule. Within each session, participants played 30 games (3 blocks of 10 - see below), with random rematching between games. ${ }^{19}$ We varied the order of the blocks between sessions (see Table 1). ${ }^{20}$ At the end of the experiment, one game was randomly chosen to be paid. The exchange rate was 1 token $=0.30$ EUR. Including a 3 EUR participation fee, earnings ranged from 3 EUR to 27 EUR, with an average of 12 EUR. The experimental software was programmed using z-Tree (Fischbacher, 2007). The experiment was conducted at the University of the Basque Country in

changed the design as described here. See Miller et al (2015) for the results of our previous experiment, which are qualitatively similar to those reported here.

${ }^{18}$ The assignment of disagreement values and initial proposer identities used in each block was generated prior to the experiment and implemented identically in all sessions. This ensured that observations are comparable across treatments and matching groups.

${ }^{19}$ Since individual $r$ values remained fixed within a given block, the matching scheme needed to guarantee that the resulting constellation of $r$ values remained fixed. In addition, we wanted to minimize repeated interaction. Therefore we required that no three-subject group is identically repeated, and no two subjects are matched more than twice in a given block. (The latter is unavoidable.) An algorithm was programmed to randomly generate a matching scheme satisfying these constraints. (Subjects were not informed about the details of this procedure.)

${ }^{20}$ Although there are six possible block orders, we only implemented five due to lack of availability of experimental subjects. 
Bilbao. Participants were undergraduate students of economics and business.

TABLE 1. SESSIONS, DECISION RULES AND BLOCK ORDERS

\begin{tabular}{l|l|c|c|c|c|c}
\hline & games & session 1 & session 3 & session 5 & session 7 & session 9 \\
\hline majority & $1-10$ & $(20,20,20)$ & $(20,20,20)$ & $(0,0,60)$ & $(0,0,60)$ & $(0,20,40)$ \\
rule & $11-20$ & $(0,20,40)$ & $(0,0,60)$ & $(0,20,40)$ & $(20,20,20)$ & $(20,20,20)$ \\
& $21-30$ & $(0,0,60)$ & $(0,20,40)$ & $(20,20,20)$ & $(0,20,40)$ & $(0,0,60)$ \\
\hline & games & session 2 & session 4 & session 6 & session 8 & session 10 \\
\hline unanimity & $1-10$ & $(20,20,20)$ & $(20,20,20)$ & $(0,0,60)$ & $(0,0,60)$ & $(0,20,40)$ \\
rule & $11-20$ & $(0,20,40)$ & $(0,0,60)$ & $(0,20,40)$ & $(20,20,20)$ & $(20,20,20)$ \\
& $21-30$ & $(0,0,60)$ & $(0,20,40)$ & $(20,20,20)$ & $(0,20,40)$ & $(0,0,60)$ \\
\hline
\end{tabular}

\subsection{Hypotheses}

As indicated, our experimental games involve $n=3$ subjects and two possible values for $q: q=3$ (unanimity rule) and $q=2$ (majority rule). The continuation probability was $\delta=\frac{2}{3}$ in all cases and three different combinations of disagreement values occurred in our experiment. For these three different combinations, Table 2 presents the equilibrium values for $z_{i}$ (player $i$ 's continuation value and the 'price' for his vote), $y_{i}$ (player $i$ 's expected equilibrium payoff) and $\mu_{i}$ (the probability of being in the final coalition as a proposer or as a coalition partner).

TABle 2. Equilibrium PREDictions

\begin{tabular}{l|ccc|cc}
\hline $\begin{array}{l}\text { Disagreement } \\
\text { values }\end{array}$ & \multicolumn{3}{|c}{ Majority rule } & \multicolumn{2}{c}{ Unanimity rule } \\
\hline$\left(r_{1}, r_{2}, r_{3}\right)$ & $\left(z_{1}, z_{2}, z_{3}\right)$ & $\left(\mu_{1}, \mu_{2}, \mu_{3}\right)$ & $\left(y_{1}, y_{2}, y_{3}\right)$ & $\left(z_{1}, z_{2}, z_{3}\right)$ & $\left(y_{1}, y_{2}, y_{3}\right)$ \\
\hline$(20,20,20)$ & $(29,29,29)$ & $\left(\frac{2}{3}, \frac{2}{3}, \frac{2}{3}\right)$ & $(33,33,33)$ & $(29,29,29)$ & $(33,33,33)$ \\
$(0,20,40)$ & $(28,29,29)$ & $\left(1, \frac{2}{3}, \frac{1}{3}\right)$ & $(43,34,24)$ & $(9,29,49)$ & $(13,33,53)$ \\
$(0,0,60)$ & $(25,25,37)$ & $\left(\frac{5}{6}, \frac{5}{6}, \frac{1}{3}\right)$ & $(38,38,25)$ & $(9,9,69)$ & $(13,13,73)$ \\
\hline
\end{tabular}

Note: $r_{i}$ is $i$ 's disagreement value; $z_{i}$ is $i$ 's acceptance threshold; $\mu_{1}$ is $i$ 's inclusion probability under majority rule; $y_{1}$ is $i$ 's expected payoff.

Our aim in the experiment will not be to test the precise point predictions presented in Table 2. Instead we will be interested in the following qualitative predictions concerning the relationship between disagreement values, probabilities of being in the coalition, offers, and expected payoffs. Moreover, we will be especially interested in comparing these relationships under majority vs. unanimity rule.

Hypothesis 1. Under both majority and unanimity rule, agreement occurs immediately (i.e. the first proposal passes).

Hypothesis 2. In the symmetric situation $r=(20,20,20)$, 
(a) Proposers build minimal winning coalitions. (They make positive offers to both responders under unanimity and to one responder under majority rule.)

(b) Offers made to coalition members do not differ between majority and unanimity rule.

Hypothesis 3. When disagreement values differ and unanimity rule is used, both responders are included and

(a) Offers received are increasing in responder disagreement values.

(b) Acceptance thresholds are increasing in responder disagreement values.

(c) Ex ante expected payoffs are increasing in disagreement values.

Hypothesis 4. When disagreement values differ and majority rule is used, one responder is included and

(a) Responders with larger disagreement values are less often included.

(b) Acceptance thresholds are weakly increasing in disagreement values.

(c) Ex ante expected payoffs are decreasing in disagreement values.

\section{Results}

Throughout our empirical analysis, we will focus exclusively on first round proposals. For each constellation of disagreement values and first round proposer identities, we investigate the distribution of proposals made, the fraction of proposals that pass, as well as the voting decisions observed. Final outcomes (i.e. agreements or breakdown that occur after round 1) will be considered only when we calculate average realized payoffs. ${ }^{21}$

\subsection{Aggregate rate of passage}

We start by considering the rate of passage. Focusing on the first round of bargaining, Figure 1 shows the fraction of proposals that pass immediately in all treatments. All values are far from 100\%, contradicting Hypothesis 1. We also observe a significantly lower rate of passage under unanimity rule, regardless of the situation. In the symmetric $(20,20,20)$ situation, the passage rate drops from

\footnotetext{
${ }^{21}$ We pool the data from all 30 periods. In the Online Appendix, we replicate all the figures reported in the paper restricting to the last 20 and 10 periods. Additionally, we replicate all the statistical analysis for the last 20 periods.
} 
$77 \%$ to $46 \%$. In the asymmetric situations, the effect is even more pronounced. Here, the passage rate drops from $78 \%$ to $30 \%$ in the $(0,20,40)$ condition, and from $79 \%$ to $38 \%$ in the $(0,0,60)$ condition. $^{22}$

Using a Mann-Whitney-Wilcoxon rank-sum test, we can establish that all of these differences are highly significant. ${ }^{23}$ For each of the three comparisons $p<$ 0.01. ${ }^{24}$ We also observe that the passage rate is very similar across situations under majority rule. In contrast, the passage rate under unanimity is significantly lower in the asymmetric situations than in the symmetric one. Using a Wilcoxon matched-pairs signed-ranks test, we can establish that the passage rate pooling the two asymmetric situations is significantly different from the passage rate in the symmetric situation $(p<0.05) .{ }^{25}$

Result 1. In all situations, passage rates are significantly lower under unanimity than under majority rule. Under unanimity rule, passage rates are lower in asymmetric situations than in the symmetric situation.

The difference in passage rates between majority and unanimity is consistent with a model of imperfect best response (see Nunnari and Zapal (2016)). Suppose players do not perfectly best respond to the strategies of others; instead, they may make errors, but they are less likely to make errors if errors are more costly. Our data would be (at least qualitatively) consistent with imperfect best response if,

\footnotetext{
${ }^{22}$ As noted by a reviewer, these effects are very large when compared to those observed in Miller and Vanberg $(2013,2015)$, who conduct 'standard' BF games with discount factors of $\delta=.9$ and $\delta=.5$. There, the passage rates under majority vs. unanimity rule are $87 \%$ vs. $70 \%$ and $88 \%$ vs. $74 \%$, respectively. Thus, the mere presence of disagreement values appears to significantly increase the amount of delay as well as the observed effect of the decision rule.

${ }^{23}$ Throughout the paper, Mann-Whitney-Wilcoxon rank-sum test and Wilcoxon matched-pairs signed-ranks test are used. We average all observations for a given matching group and use matching group averages as the unit of observation. All p-values reported are two-tailed and have been calculated using the exact values as proposed by Harris and Hardin (2013).

${ }^{24}$ Delay under unanimity rule would be compatible with subjects playing a subgame perfect equilibrium (SPE) with history-dependent strategies rather than an SSPE (see Herings et al., 2017). However, some features of behavior are incompatible with even SPE. Consider a proposal that is rejected by only one player. Because the player could have accepted the proposal (and, since this would result in the end of the game, such deviation cannot be punished by other players), the expected payoff from rejecting the proposal cannot be lower than the payoff offered in the proposal. Our data does not satisfy this restriction for subjects with low disagreement values: on average, being the sole rejector results in a slight loss when $r_{i}=20$, and a substantial loss when $r_{i}=0$. This finding is consistent with the high frequency of disadvantageous counterproposals found in earlier experiments on two-person bargaining (see Ochs and Roth, 1989).

${ }^{25}$ Pairwise comparisons of the three conditions tell a similar story, although not all comparisons are statistically significant: $p<0.05$ for $(20,20,20)$ vs. $(0,20,40) ; p=0.13$ for $(20,20,20)$ vs. $(0,0,60) ; p=0.10$ for $(0,20,40)$ vs. $(0,0,60)$.
} 

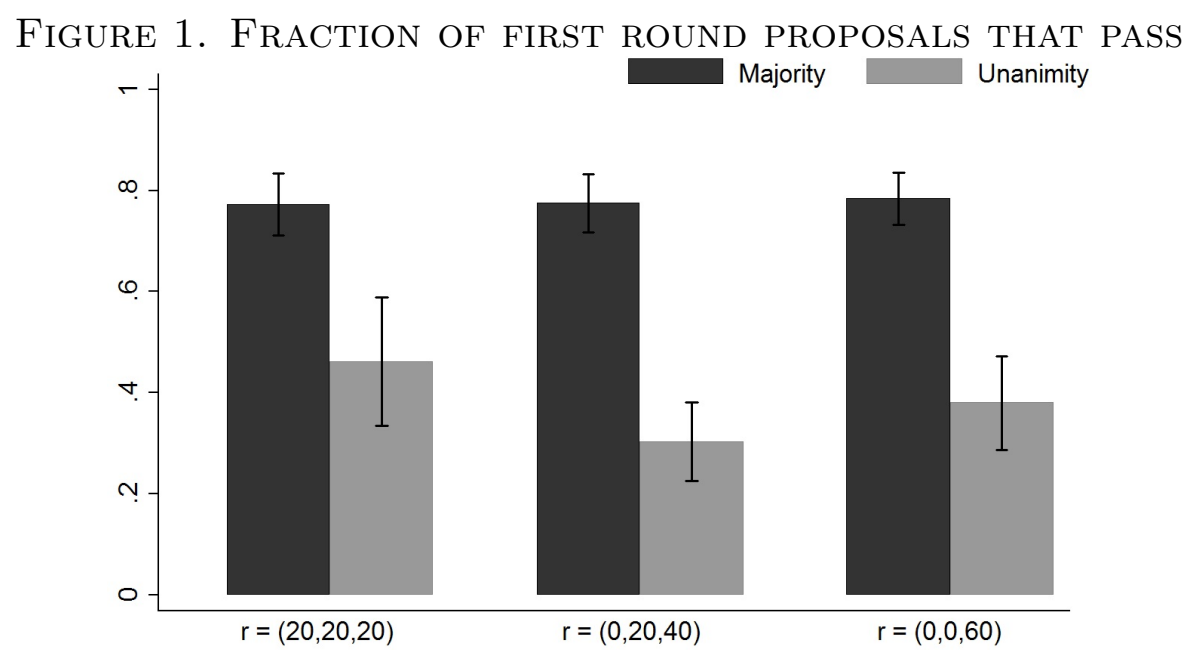

Notes: The fractions of first round proposals that pass, pooling all the periods, are graphed as vertical bars; the whiskers indicate $95 \%$ confidence intervals. Confidence intervals are calculated using an average in each matching group to account for interdependencies between observations that come from the same matching group.

given the actual behavior in the experiment, playing a strategy that causes delay is more costly under majority than under unanimity.

Let $p$ be the probability that an agreement is reached in any given round (assume $p$ is the same for all rounds). We can compute the probability that an agreement is reached before negotiations break down as follows. Agreement in the first round occurs with probability $p$. Agreement in the second round occurs if, after players fail to reach an agreement in the first round (this occurs with probability $1-p$ ), negotiations do not break down, such that the game moves to round 2 (this occurs with probability $\delta$ ), and then players agree (with probability $p)$. Iterating this reasoning, the overall probability that an agreement is reached before breakdown occurs is $p+(1-p) \delta p+(1-p) \delta(1-p) \delta p+\ldots=\frac{p}{1-(1-p) \delta}$.

In our experiment we have $\delta=\frac{2}{3}$. Consider the symmetric case, $r=(20,20,20)$. The passage rate we observe under unanimity is 0.46. If we assume a constant probability of agreement $p=0.46$ in each round, the overall probability of agreement is $\frac{0.46}{1-0.54 * 2 / 3}=\frac{23}{32}$. Expected payoffs, calculated at the beginning of a round before Nature selects a proposer, are $\frac{23}{32} * \frac{100}{3}+\left[1-\frac{23}{32}\right] * 20=29.58$ for each player (we are implicitly assuming that strategies are stationary and symmetric, such that each player expects $\frac{100}{3}$ if agreement is reached). The empirical continuation value under unanimity is then $\frac{2}{3} * 29.58+\frac{1}{3} * 20=26.39$ (breakdown occurs immediately with probability $\frac{1}{3}$, in which case players get 20; negotiations continue with probability $\frac{2}{3}$, in which case players may eventually reach an agreement or nor as discussed above). Under majority, given the passage rate $p=0.77$ observed 
in our experiment, we obtain an empirical continuation value of 28.08.

Under majority, the proposer could propose $(50,50,0)$, and this would give him and one other player almost 22 points above their continuation value; similarly, if the proposer proposes $(60,40,0)$, this would still give the intended coalition partner almost 12 points above the continuation value. Because the proposer has a lot to lose from making a proposal that would likely be rejected, and the coalition partner has a lot to lose from rejecting a proposal like $(50,50,0)$ or even $(60,40,0)$, we expect that players will often come to an agreement.

Under unanimity, all players have to agree in order for a proposal to pass, hence they would all need to be given a payoff well above their continuation value (the responders in order to have an incentive to vote yes, the proposer in order to have an incentive to make the proposal in the first place). Even if the proposer proposes $(34,33,33)$, the gain would be $34-26.39=7.61$ for the proposer and $33-26.39=6.61$ for each responder. Hence, compared with the majority case, errors that lead to delay are clearly less costly under unanimity, and we would expect delay to occur much more often, as it is the case. ${ }^{26}$

\subsection{Symmetric situation $r=(20,20,20)$}

Figure 2 depicts, within a unit simplex, the frequency of first round proposals (bubble size) and the corresponding passage rates (pie charts within bubbles) in the symmetric $r=(20,20,20)$ condition. The data are formatted such that the first coordinate corresponds to the proposer's share. The second and the third coordinates correspond to the responders' shares. When responder $r_{i}$ values are the same, they are ordered alphabetically. For example, suppose that player ' $\mathrm{C}$ ' is the proposer. Then the first coordinate is $x_{C}$, the second is $x_{A}$, and the third is $x_{B}$. Given this formatting, the bottom left, right, and top left corners of the simplex represent points at which the entire pie is allocated to the proposer, responder 1, and responder 2, respectively. Finally, each figure contains a set of reference points. Our theoretical predictions are marked by red dots, and all equal splits (two-way and three-way) are marked by blue dots.

The left panel (a) shows proposals and passage rates under majority rule and the right panel (b) under unanimity rule. In addition, each panel identifies the

\footnotetext{
${ }^{26}$ One may ask if the higher delay under unanimity relative to the earlier experiments of Miller and Vanberg $(2013,2015)$ is consistent with imperfect best response. Let $p$ be the passage rate, and $\delta$ the discount factor. The continuation value is then $\delta p \frac{100}{3}+\delta(1-p) \delta p \frac{100}{3}+\ldots=\frac{\delta p \frac{100}{3}}{1-\delta(1-p)}$. Given the observed passage rates of $p=74 \%$ for $\delta=0.5$ and $p=70 \%$ for $\delta=0.9$, the continuation values are 14.18 and 28.77 respectively. Our data are consistent with those for $\delta=0.5$ in the sense that delay is both much less costly and much more often observed. The data for $\delta=0.9$ are out of line with the other two experiments, since they contain both the highest continuation value and a very high passage rate.
} 
Figure 2. Proposals And Passage Rates, $r=(20,20,20)$

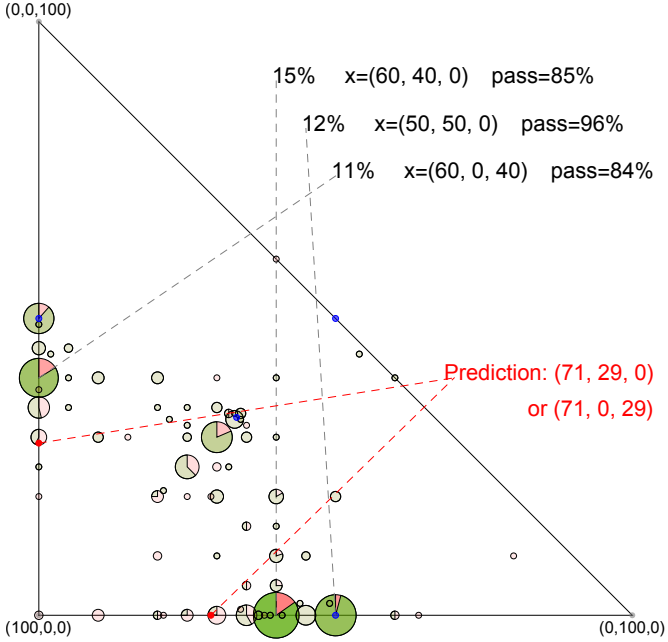

(a) Majority rule $(\mathrm{N}=400)$

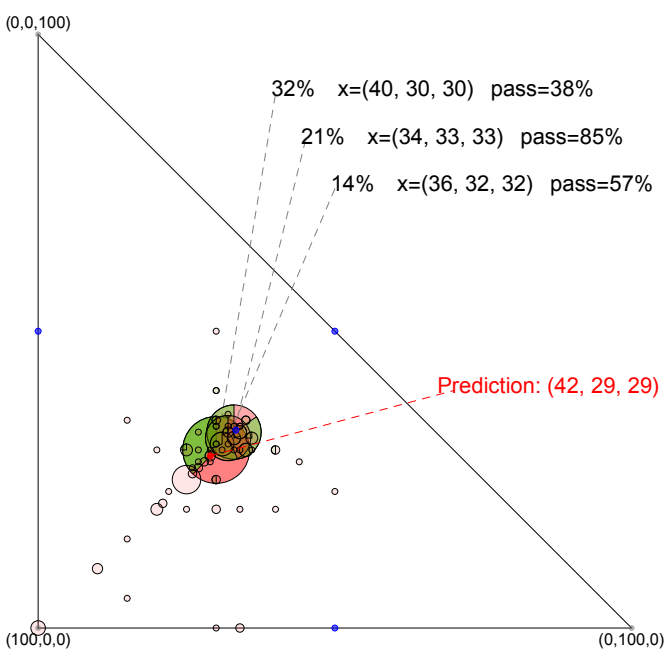

(b) Unanimity rule $(\mathrm{N}=400)$

three most frequent proposals and their corresponding passage rate. For instance, the modal proposal under majority rule is $x=(60,40,0)$, and this proposal passes $85 \%$ of the time. Similarly, the modal proposal under unanimity rule is $x=$ $(40,30,30)$, and is passed only $38 \%$ of the time.

As can be seen in panel (a), most proposals under majority rule are located along the edge of the simplex, meaning that the proposer is attempting to build a minimal winning coalition with only one responder receiving a positive share $(65 \%$ overall, $85 \%$ in the final ten periods). In panel (b), all but 9 out of 400 proposals allocate positive shares to both responders. Taken together, these numbers support Hypothesis 2a concerning the size of proposed coalitions.

Result 2a. In the symmetric situation $r=(20,20,20)$, most proposers attempt to build a minimal winning coalition.

Under majority rule, the most frequent proposals are those that allocate $40 \%$ to one of the responders (26\% of proposals are of this type, $29 \%$ in the last ten periods). Interestingly, these modal offers are located approximately half way between the two-way equal split and the equilibrium prediction, which allocates $29 \%$ to a single responder. Under unanimity rule, the modal proposal is $x=(40,30,30)$, which is remarkably close to our equilibrium prediction $(x=(42,29,29))$. Overall, proposals under unanimity rule are concentrated in the area between our equilibrium prediction and the three-way equal split. These patterns are consistent with findings from the previous literature. 
Based on a preliminary inspection of Figure 2, it appears that Hypothesis $2 \mathrm{~b}$ (equal offers under both rules) can be rejected. In particular, the modal proposal under majority rule is one where a single coalition partner is offered $40 \%$ of the pie. Under unanimity rule, in contrast, there are virtually no proposals which allocate more than $33 \%$ to a coalition partner. This suggests that (positive) offers under majority rule tend to be larger than those made under unanimity rule. And indeed, if we pool the data from all periods and sessions, we find that coalition partners under majority rule are offered on average 4 tokens more than coalition partners under unanimity rule (34.6 vs. 30.4). This pattern is consistent with imperfect best response as discussed earlier.

Using a Mann-Whitney-Wilcoxon rank-sum test, we conclude that this difference is significant $(p<0.0001)$. The same conclusion emerges when we conduct a regression analysis. We estimate the effect of the decision rule on offers to coalition partners, using a random effects linear regression. ${ }^{27}$ The results of this analysis are reported in the first column of Table 3. Offers increase with the period and are significantly smaller under unanimity rule. Thus we reject Hypothesis $2 \mathrm{~b}$.

Result 2b. In the symmetric situation $r=(20,20,20)$, coalition partners (defined as those who are offered positive shares) are offered more under majority rule than under unanimity rule.

As we know from Figure 1, and in line with previous experiments without disagreement values, most proposals pass under majority rule $(77 \%$ overall, $74 \%$ in the last ten periods). In contrast, proposals under unanimity rule often fail, with only $46 \%$ passing in the first bargaining round ( $49 \%$ in the last ten periods). Under unanimity rule, only three-way equal splits $(x=(34,33,33))$ are consistently passed (85\%), and even small deviations from such an equal split result in high rates of failure. For instance, the third most frequent proposal, $x=(36,32,32)$, passes in only $57 \%$ of cases. The modal offer (corresponding to the equilibrium prediction) passes only $38 \%$ of the time.

To understand the voting behavior underlying these different passage rates, we turn to Figure 3. Here, the pie charts within bubbles represent the votes cast by responder 1 (left panel) and responder 2 (right panel). The green and red areas represent the fraction of 'yes' and 'no' votes, respectively.

Under majority rule, responders included in a minimal winning coalition usually vote yes (see the prevalence of green bubbles in the horizontal and vertical axes

\footnotetext{
${ }^{27}$ Specifically, we estimate the following equation: $x_{i t}=\beta_{0}+\beta_{1} U_{i}+\beta_{2} P_{t}+\sum_{j=2}^{5} \beta_{(1+j)} O_{i}+$ $\alpha_{i}+\nu_{i t}$, where we estimate the effect of the unanimity rule $\left(U_{i}\right)$ on the offer to coalition partners $\left(x_{i t}\right)$, controlling for a linear effect of the period $\left(P_{t}\right)$ and the block order $\left(O_{i}\right)$, which is introduced in the equation as a set of four dummies; $\alpha_{i}$ and $\nu_{i t}$ are a subject specific and an idiosyncratic error term, respectively.
} 
Figure 3. Voting Behavior, $r=(20,20,20)$

Majority Rule (N=400)

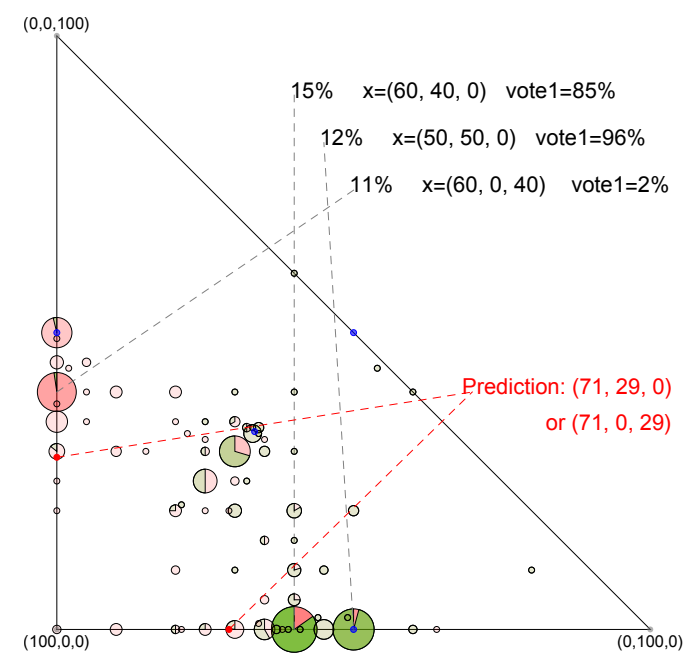

(a) Responder 1's vote

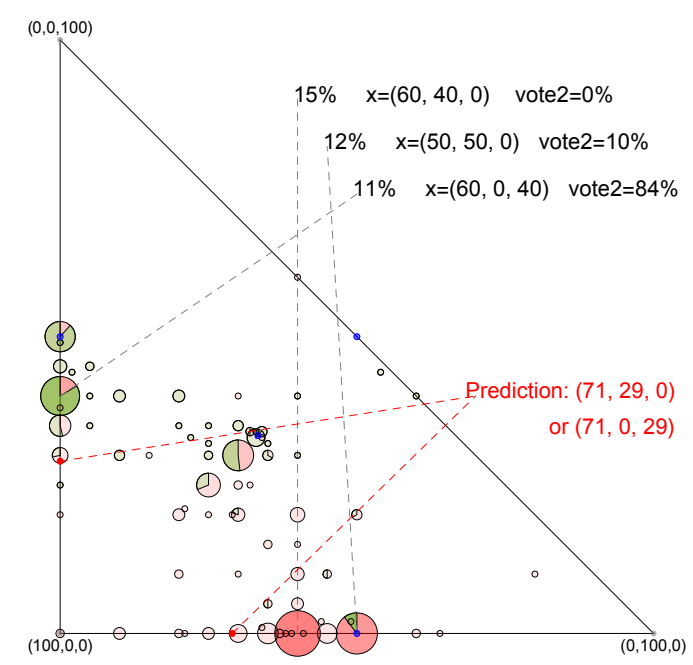

(b) Responder 2's vote

Unanimity Rule $(\mathrm{N}=400)$

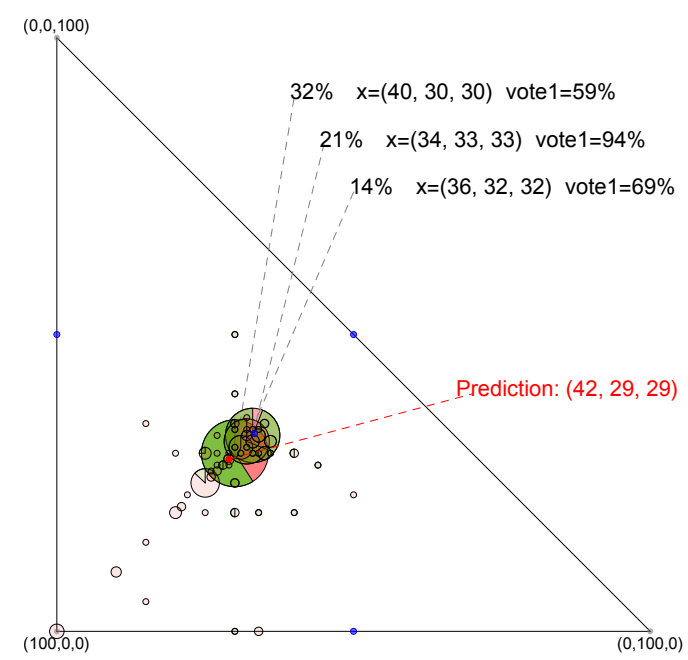

(c) Responder 1's vote

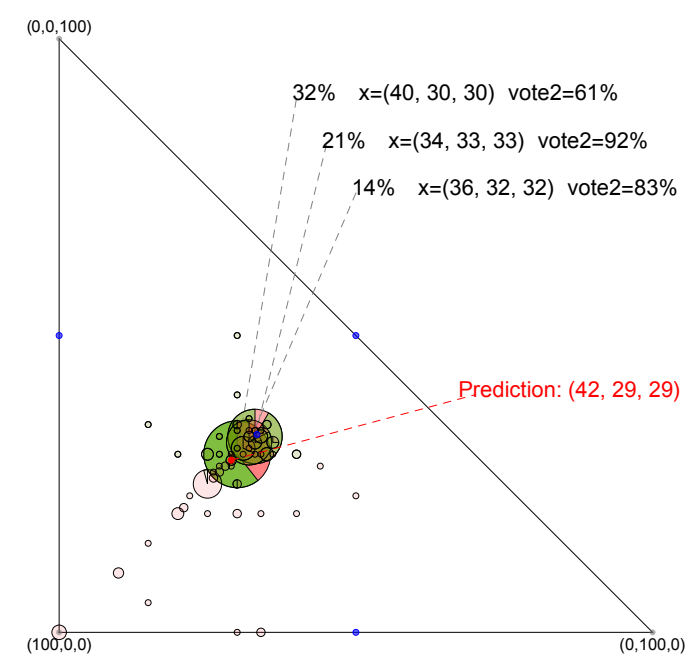

(d) Responder 2's vote 
of panels (a) and (b), respectively) and the excluded responder votes no (see the prevalence of red bubbles in the vertical and horizontal axes of panels (a) and (b), respectively). This pattern explains the relatively high rate of passage under majority rule. Under unanimity rule (panels (c) and (d)), we see that responders vote yes on the equal split $94 \%$ and $92 \%$ of the time. In contrast, the 'almost equal' split $x=(36,32,32)$ is accepted by only $69 \%$ and $83 \%$ of voters 1 and 2 , respectively, resulting in a passage rate of only $57 \%$. Finally, the modal proposal $(40,30,30)$ is accepted by only $59 \%$, resp. $61 \%$ of responders 1 and 2 . Since one no vote suffices to reject such a proposal, this explains the low passage rates under unanimity rule.

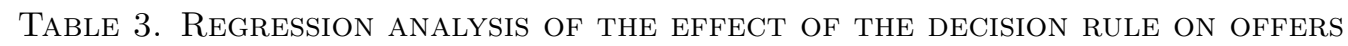
TO COALITION PARTNERS

\begin{tabular}{|c|c|c|c|c|c|}
\hline \multicolumn{6}{|c|}{$\begin{array}{l}\text { Dependent variable: "Offer to coalition partner" } \\
\text { Participant random effects included in all models }\end{array}$} \\
\hline & $\begin{array}{l}\text { Symmetric } r \\
\text { Pooled }\end{array}$ & $\begin{array}{l}r=(0,20,40) \\
\text { Unanimity }\end{array}$ & $\begin{array}{c}r=(0,20,40) \\
\text { Majority }\end{array}$ & $\begin{array}{c}r=(0,0,60) \\
\text { Unanimity }\end{array}$ & $\begin{array}{c}r=(0,0,60) \\
\text { Majority }\end{array}$ \\
\hline Unanimity & $\begin{array}{c}-4.87^{* * *} \\
(0.69)\end{array}$ & & & & \\
\hline Period & $\begin{array}{c}0.27^{* * *} \\
(0.07)\end{array}$ & $\begin{array}{l}0.16^{*} \\
(0.08)\end{array}$ & $\begin{array}{l}-0.20 \\
(0.18)\end{array}$ & $\begin{array}{c}0.30^{* *} \\
(0.14)\end{array}$ & $\begin{array}{c}0.21 \\
(0.19)\end{array}$ \\
\hline$R r=20$ & & $\begin{array}{c}8.60^{* * *} \\
(0.62)\end{array}$ & $\begin{array}{c}0.92 \\
(1.58)\end{array}$ & & \\
\hline$R r=40$ & & $\begin{array}{l}21.91^{* * *} \\
(0.61)\end{array}$ & $\begin{array}{c}0.20 \\
(1.59)\end{array}$ & & \\
\hline$R r=60$ & & & & $\begin{array}{c}30.65^{* * *} \\
(0.92)\end{array}$ & $\begin{array}{l}-1.83 \\
(1.44)\end{array}$ \\
\hline $\operatorname{Pr}=20$ & & $\begin{array}{c}-1.45^{* *} \\
(0.73)\end{array}$ & $\begin{array}{l}-2.63 \\
(1.87)\end{array}$ & & \\
\hline $\operatorname{Pr}=40$ & & $\begin{array}{c}-2.35^{* * *} \\
(0.73)\end{array}$ & $\begin{array}{c}-3.67^{* *} \\
(1.80)\end{array}$ & & \\
\hline $\operatorname{Pr}=60$ & & & & $\begin{array}{c}-2.96^{* * *} \\
(1.08)\end{array}$ & $\begin{array}{c}-6.13^{* * *} \\
(1.63)\end{array}$ \\
\hline Constant & $\begin{array}{c}33.18^{* * *} \\
(0.93)\end{array}$ & $\begin{array}{c}19.69^{* * *} \\
(1.56)\end{array}$ & $\begin{array}{c}38.26^{* * *} \\
(3.63)\end{array}$ & $\begin{array}{c}14.36^{* * *} \\
(3.71)\end{array}$ & $\begin{array}{c}27.09^{* * *} \\
(5.11)\end{array}$ \\
\hline Order dummies & Yes & Yes & Yes & Yes & Yes \\
\hline Observations & 1,325 & 794 & 516 & 772 & 549 \\
\hline Number of id & 240 & 120 & 120 & 120 & 120 \\
\hline
\end{tabular}

Notes: Sample includes offers to coalition partners (positive offers); $R r$ and $P r$ are responder's and proposer's $r$ value, respectively; standard errors in parentheses; ${ }^{* *}$ - sig. at $1 \%$;

** - sig. at $5 \% ;{ }^{*}$ - sig. at $10 \%$.

\subsection{Asymmetric $r$ : Unanimity rule}

Figures 4 and 5 summarize the frequency of proposals and the passage rates under unanimity rule in the asymmetric conditions $(r=(0,20,40)$ and $r=(0,0,60))$. 
Figure 4. Proposals and passage Rates, $r=(0,0,60)$, UNANIMITY RULE $(\mathrm{N}=400)$

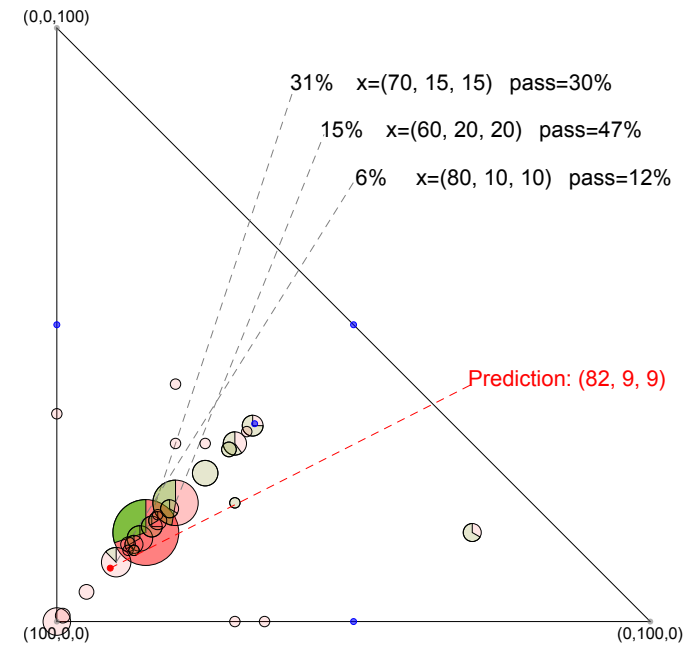

(a) $r=(60,0,0)(\mathrm{N}=130)$

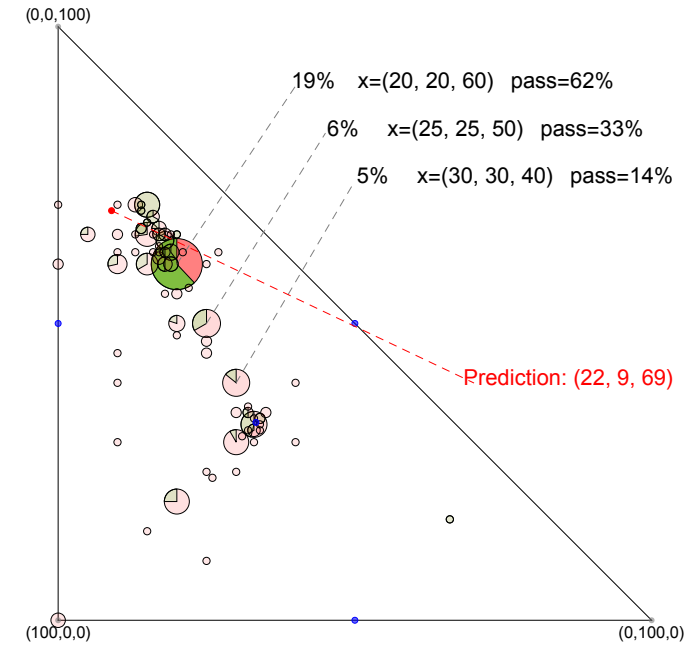

(b) $r=(0,0,60)(\mathrm{N}=270)$

Within each condition, the figures distinguish according to the proposer's $r_{i}$ value. The $r$ vector below each diagram, as well as the points plotted, are defined such that the first coordinate corresponds to the proposer's disagreement value and allocated share, and the second and third coordinates those of responders 1 and 2, respectively. When responder disagreement values differ, responder 1 is the one with the lower disagreement value. When they are the same, responders are alphabetically ordered. As above, each panel identifies the three most frequent proposals in each situation. For example, Figure 4(a) depicts proposals made by the player with $r_{i}=60$. In Figure 4(b), the proposer's $r_{i}=0$, the first responder (right corner) has $r_{i}=0$, and the second responder (top corner) has $r_{i}=60$.

As in the symmetric situation, it appears that proposals are concentrated around a line connecting the three-way equal split (marked in blue) and the equilibrium prediction (marked in red). For example, in figure 4, panel (a), the equilibrium prediction is $x=(82,9,9)$ and the modal proposal is $x=(70,15,15)$. Most proposals are closer to the prediction than the equal split. In fact, modal proposals are remarkably close to the theoretical predictions. In figure 5 (c), the modal proposal allocates to both responders one token more than what is predicted.

Two patterns are immediately visible in these figures. First, whenever responder disagreement values differ (Figure 4(b) and Figure 5), almost all observations are located above the $45^{\circ}$ line. That is, responder 2 (with the higher disagreement value) is consistently offered more. This is consistent with Hypothesis 3a. Pooling 
Figure 5. Proposals and passage rates, $r=(0,20,40)$, UNANIMITY RULE $(\mathrm{N}=400)$

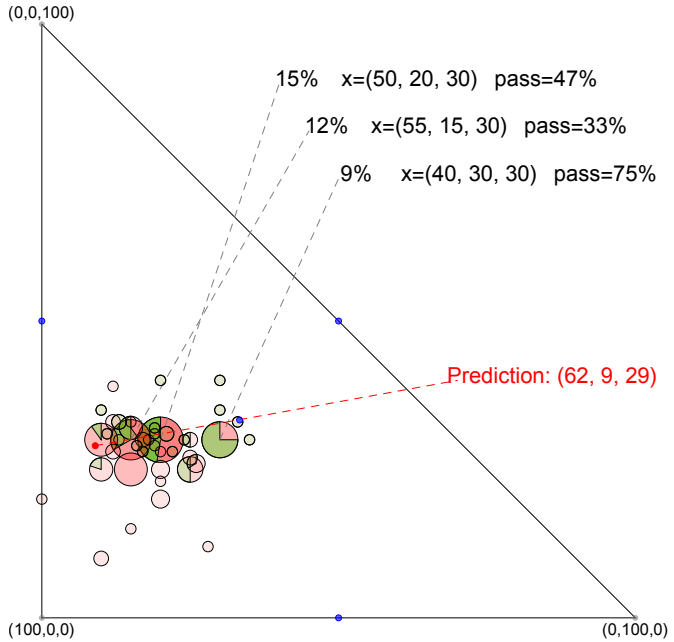

(a) $r=(40,0,20)(\mathrm{N}=130)$

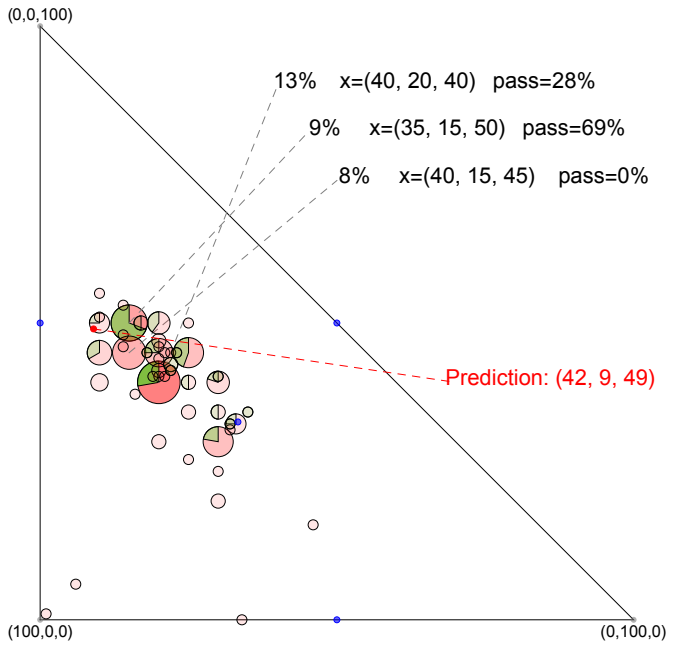

(b) $r=(20,0,40)(\mathrm{N}=140)$

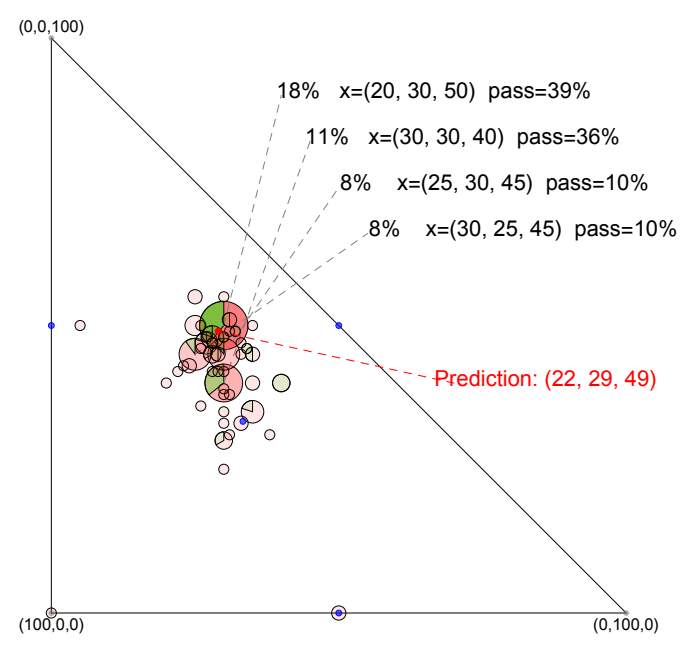

(c) $r=(0,20,40)(\mathrm{N}=130)$ 
the data from all periods for each of the asymmetric conditions (i.e. combining the observations displayed in Figures 4 and Figure 5), we find that average offers received are increasing in responder disagreement values. In the $r=(0,0,60)$ condition, responders with $r_{i}=0$ and $r_{i}=60$ are offered an average of 19 and 51 tokens, respectively. (Note, however, that the latter offer is lower than the disagreement value, which is not consistent with our theory.) In the $r=(0,20,40)$ condition, responders are offered 20,29, and 42 tokens on average. ${ }^{28,29}$

The significance of these differences is confirmed by regression analysis. We extend the analysis reported in the previous section to test whether offers received are positively related to responder disagreement values. ${ }^{30}$ Results are reported in columns 2 and 4 of table 3 . As predicted, offers are monotonically increasing in responder $r$ values under unanimity rule. (Offers also decrease slightly as proposer's disagreement value increases, similarly to Diermeier and Gailmard's (2006) findings for the ultimatum game. This effect is not predicted by the theory.)

Result 3a. In asymmetric situations and under unanimity rule, offers received are increasing in responder disagreement values.

A second pattern discernible from Figures 4 and 5 is that a large fraction of proposals fail. The average passage rate for all periods and asymmetric constellations under unanimity rule is only $34 \%$ (38\% in the last ten periods). This passage rate is slightly higher when $r=(0,0,60)(38 \%)$ than when $r=(0,20,40)(30 \%)$.

We can get a feel for the voting behavior underlying these passage rates by looking at Figures 6 and 7, which are constructed analogously to Figure 3. When their disagreement values differ, visual inspection suggests that responder 2 (with the larger disagreement value) more often votes against the proposal. Furthermore, this happens despite the fact that responder 2 is consistently offered more. Although we cannot observe acceptance thresholds directly, this pattern is consistent with the hypothesis that acceptance thresholds are increasing in disagreement values (Hypothesis 3b).

\footnotetext{
${ }^{28}$ Using a Wilcoxon matched-pairs signed-ranks test, we can establish that the amount offered to the player with the largest disagreement value is different from the amount offered to the other players $(p<0.01)$. Using the same test, we can show that, in the $r=(0,20,40)$ condition, the amount offered to $r_{i}=0$ is different from the amount offered to $r_{i}=20$ and the latter is different from the amount offered to $r_{i}=40$. All comparisons return values of $p<0.01$.

${ }^{29}$ Note that actual offers are less sensitive to disagreement values than predicted offers. This echoes the results of Anbarci and Feltovich (2013) for two-player bargaining with a deadline.

${ }^{30}$ Specifically, we estimate the following equation: $x_{i t}=\beta_{0}+\beta_{1} P_{t}+\sum_{j=2}^{4} \beta_{(0+j)} R r_{i}+$ $\sum_{k=2}^{4} \beta_{(3+k)} \operatorname{Pr}_{i}+\sum_{l=2}^{5} \beta_{(6+l)} O_{i}+\alpha_{i}+\nu_{i t}$, where we estimate the effect of responder's disagreement value $\left(R r_{i}\right)$ on offers received $\left(x_{i t}\right)$, controlling for the period, the block order, and
} 
Figure 6. Voting Behavior, $r=(0,0,60)$, UnANimity Rule $(\mathrm{N}=400)$

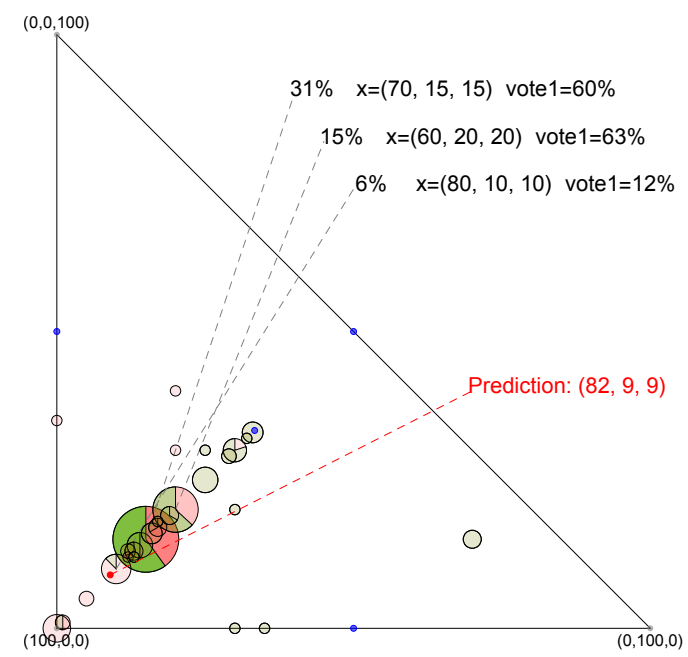

(a) $r=(60,0,0)$, Responder 1's vote

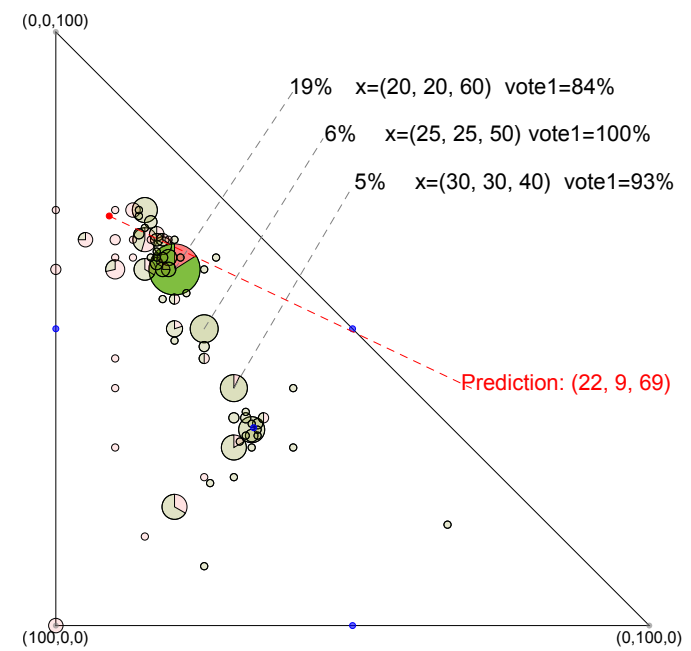

(c) $r=(0,0,60)$, Responder 1's vote

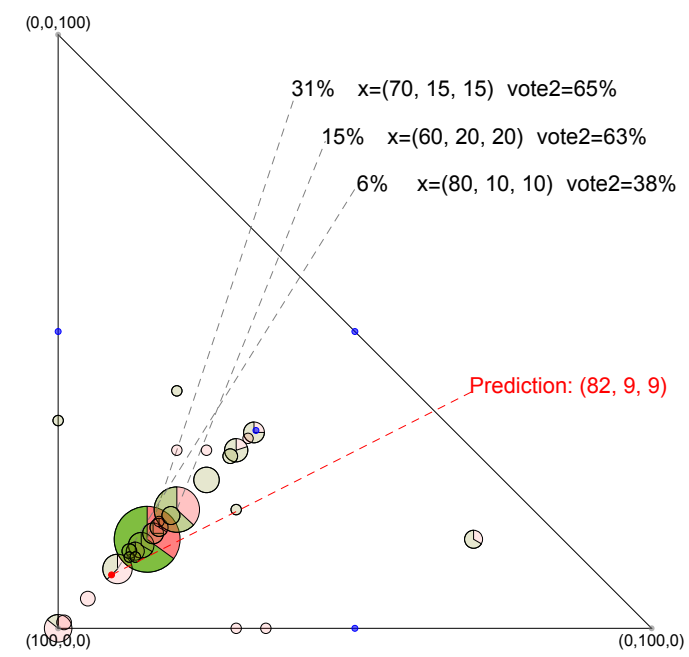

(b) $r=(60,0,0)$, Responder 2's vote

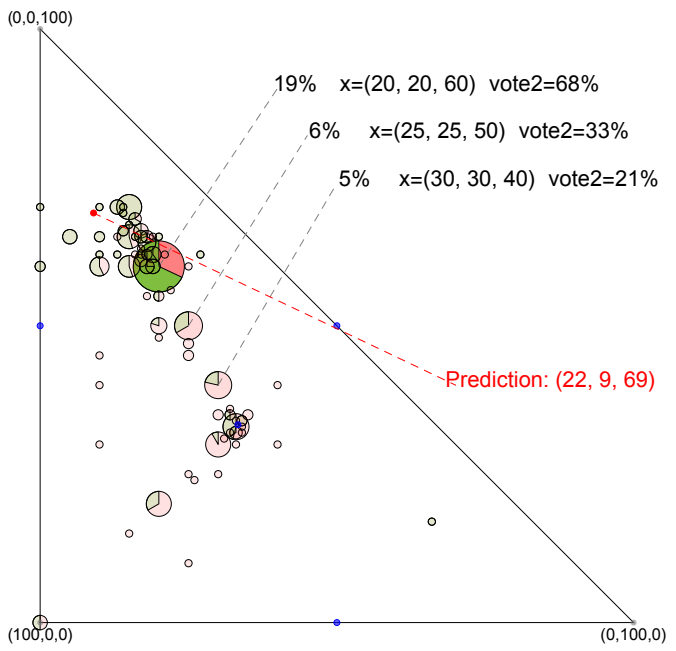

(d) $r=(0,0,60)$, Responder 2's vote 
Figure 7. Voting Behavior, $r=(0,20,40)$, UnANimity RUle $(\mathrm{N}=400)$

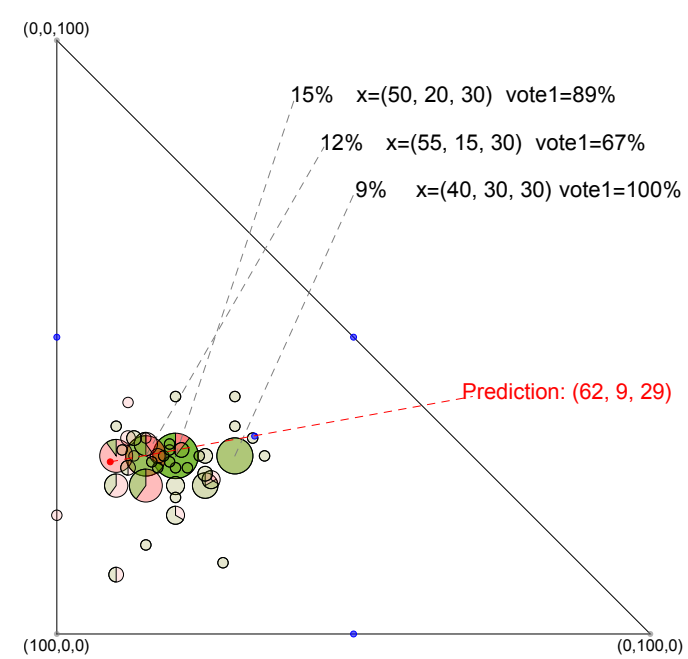

(a) $r=(40,0,20)$, Responder 1's vote

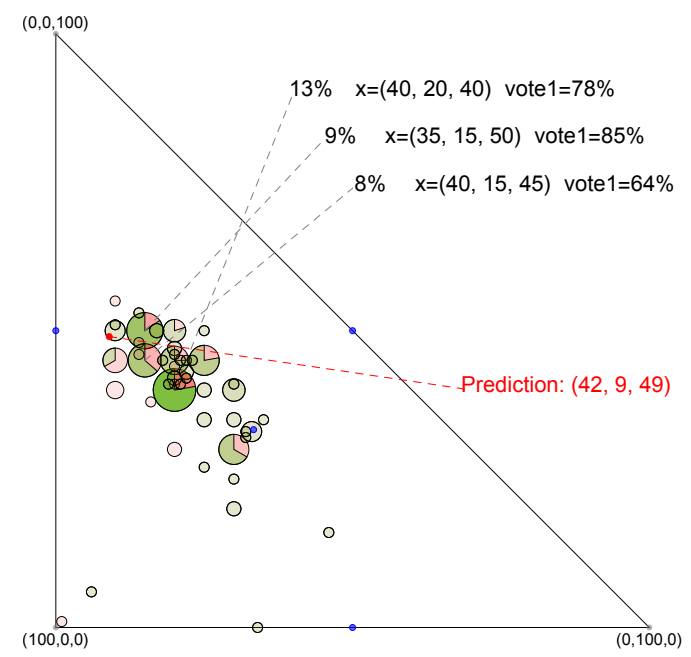

(c) $r=(20,0,40)$, Responder 1's vote

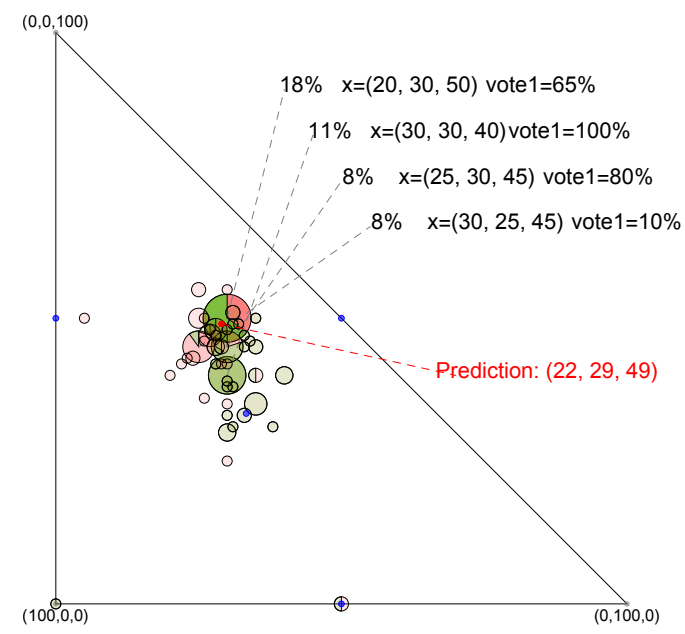

(e) $r=(0,20,40)$, Responder 1's vote

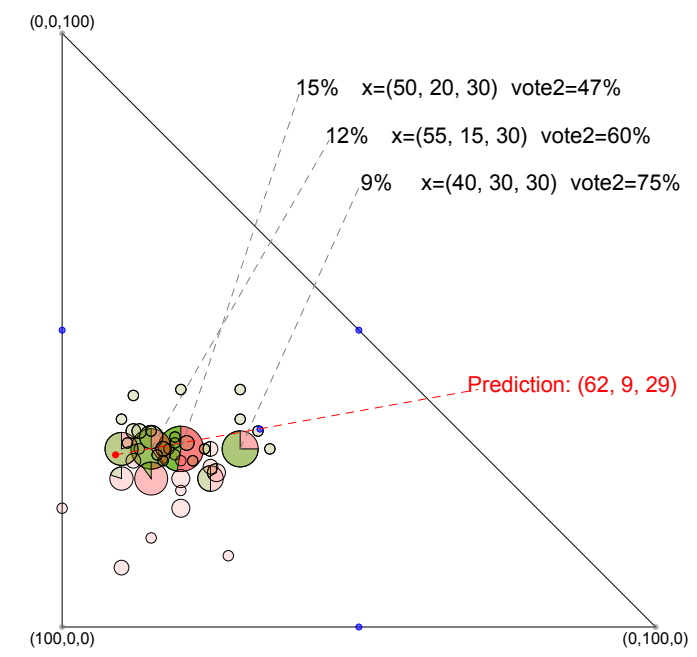

(b) $r=(40,0,20)$, Responder 2's vote

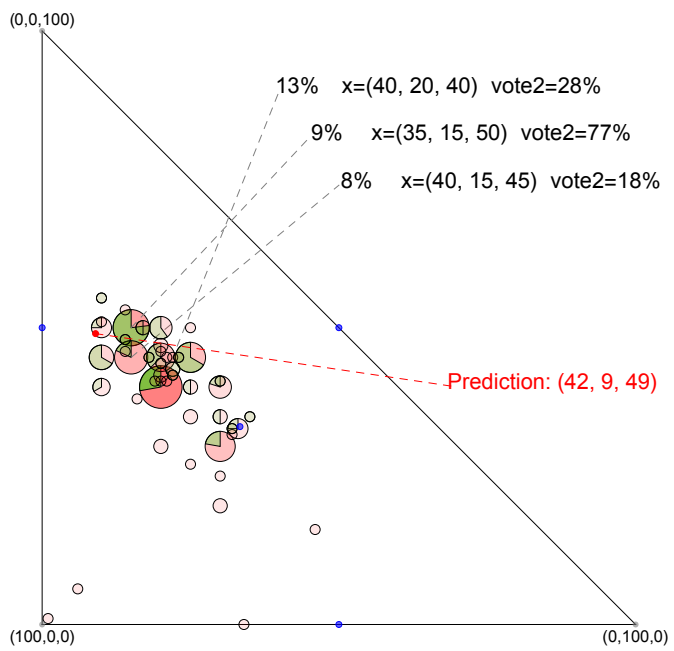

(d) $r=(20,0,40)$, Responder 2's vote

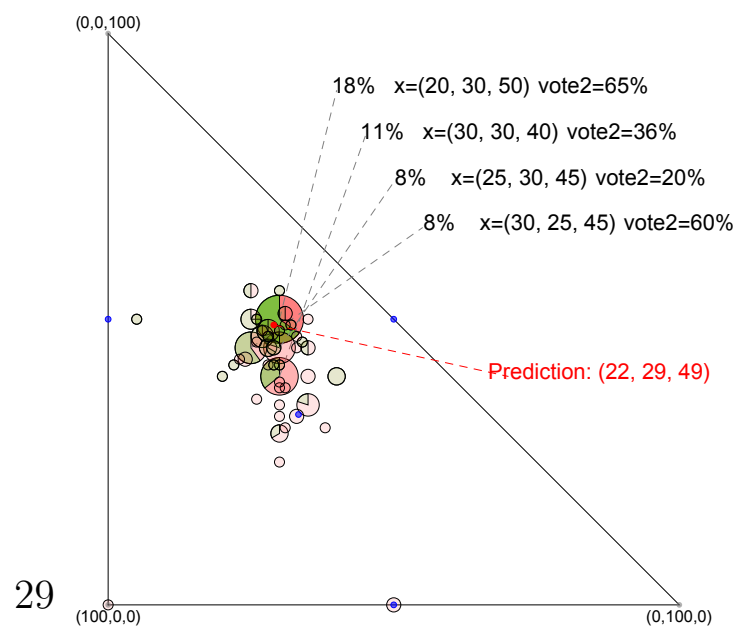

(f) $r=(0,20,40)$, Responder 2's vote 
To assess the significance of these differences in voting behavior, we conduct a regression analysis that controls for characteristics of the proposals. We estimate the effects of disagreement value on individual voting decisions, using a random effects probit regression. ${ }^{31}$ Results are reported in columns 1 and 3 of table 4 . Controlling for the kind of offer being considered, we find that the likelihood that a player votes yes is decreasing in her disagreement value.

Result 3b. In asymmetric situations and under unanimity rule, the likelihood that a player votes yes on a given offer is decreasing in her disagreement value.

the proposer's disagreement value $\left(P r_{i}\right)$. Responder's and proposer's disagreement values are introduced as sets of dummies in the regression analysis.

${ }^{31}$ Specifically, we estimate the following equation: vote $_{i t}=1\left(\beta_{0}+\beta_{1} O S_{i t}+\beta_{2} P S_{i t}+\beta_{3} P_{t}+\right.$ $\left.\sum_{j=2}^{4} \beta_{(2+j)} R r_{i}+\sum_{k=2}^{4} \beta_{(5+k)} P r_{i}+\sum_{l=2}^{5} \beta_{(8+l)} O_{i}+\nu_{i t} \geq 0\right)$, where we estimate the effect of responder's disagreement value $\left(R r_{i t}\right)$ on the vote to a proposal (vote $e_{i t}$ ), controlling for the responder's own share $\left(O S_{i t}\right)$, the proposer's share $\left(P S_{i t}\right)$, as well as the rest of controls introduced in the analysis of offers above. 
TABle 4. REgRESSION ANALYSis OF THE PROBABILITy OF VOTING YeS

\begin{tabular}{|c|c|c|c|c|}
\hline \multicolumn{5}{|c|}{$\begin{array}{l}\text { Dependent variable: "Voting yes" } \\
\text { Participant random effects included in all models }\end{array}$} \\
\hline \multicolumn{5}{|c|}{ Dependent variable: "Voting yes" (asymmetric situations) } \\
\hline & $r=(0,20,40)$ & $r=(0,20,40)$ & $r=(0,0,60)$ & $r=(0,0,60)$ \\
\hline & Unanimity & Majority & Unanimity & Majority \\
\hline \multirow[t]{2}{*}{ Own share } & $0.091^{* * *}$ & $0.094^{* * *}$ & $0.044^{* * *}$ & $0.071^{* * *}$ \\
\hline & $(0.011)$ & $(0.010)$ & $(0.006)$ & $(0.007)$ \\
\hline \multirow[t]{2}{*}{ Proposer's share } & $-0.031 * * *$ & -0.004 & $-0.013^{* *}$ & $-0.013^{* * *}$ \\
\hline & $(0.009)$ & $(0.006)$ & $(0.005)$ & $(0.005)$ \\
\hline \multirow[t]{2}{*}{ Period } & -0.026 & -0.005 & 0.017 & 0.009 \\
\hline & $(0.018)$ & $(0.026)$ & $(0.018)$ & $(0.025)$ \\
\hline \multirow[t]{2}{*}{$R r=20$} & $-1.497 * * *$ & $-0.729 * * *$ & & \\
\hline & $(0.188)$ & $(0.224)$ & & \\
\hline \multirow[t]{2}{*}{$R r=40$} & $-3.200 * * *$ & $-1.092^{* * *}$ & & \\
\hline & $(0.315)$ & $(0.237)$ & & \\
\hline \multirow[t]{2}{*}{$R r=60$} & & & $-2.201^{* * *}$ & $-0.971^{* * *}$ \\
\hline & & & $(0.241)$ & $(0.181)$ \\
\hline \multirow[t]{2}{*}{$\operatorname{Pr}=20$} & $0.736^{* * *}$ & 0.157 & & \\
\hline & $(0.279)$ & $(0.226)$ & & \\
\hline \multirow[t]{2}{*}{$\operatorname{Pr}=40$} & $-0.971^{* * *}$ & $-0.971^{* * *}$ & & \\
\hline & $(0.181)$ & $(0.181)$ & & \\
\hline \multirow[t]{2}{*}{$\operatorname{Pr}=60$} & & & 0.161 & 0.066 \\
\hline & & & $(0.239)$ & $(0.160)$ \\
\hline \multirow[t]{2}{*}{ Constant } & 0.357 & $-1.572^{* *}$ & -0.102 & -0.594 \\
\hline & $(0.479)$ & $(0.626)$ & $(0.507)$ & $(0.674)$ \\
\hline Order dummies & Yes & Yes & Yes & Yes \\
\hline Observations & 794 & 516 & 549 & 549 \\
\hline Number of id & 120 & 120 & 120 & 120 \\
\hline
\end{tabular}

Notes: Sample includes votes of coalition partners; $R r$ and $P r$ are responder's and proposer's $r$ value, respectively; marginal effects taken at variables means from random effect probit regressions presented; standard errors in parentheses; ${ }^{* * *}$ - sig. at $1 \% ;{ }^{* *}$ - sig. at $5 \%{ }^{*}$ - sig. at $10 \%$.

Interestingly, the reason for the high proportion of negative votes and subsequent proposal failures under unanimity rule is not that players with high disagreement values try to demand very high shares. Although proposers' demands are monotonic in disagreement values, the vast majority of them are substantially below of what they should demand according to theoretical predictions. As figures 2, 4 and 5 show, proposals that significantly deviate from an equal split are consistently rejected. Failures of close-to-the-equilibrium proposals are more common in asymmetric situations and when the proposer has the higher disagreement value. For instance, in the $r=(0,0,60)$ situation, proposals are almost always rejected when the proposer demand is close to the equilibrium prediction of 82 .

Finally, let us look at the payoffs realized within the asymmetric situations under unanimity rule. In Table 5, we present average final payoffs in each situation. The top part of the table distinguishes cases according to which player was initially 
assigned the proposer role. Using these numbers, the ex ante expected payoffs are computed by taking the appropriate weighted averages. These numbers are reported at the bottom of the table. Consistent with Hypothesis 3(c), the player with the largest disagreement value achieves the largest expected payoff. ${ }^{32}$

\begin{tabular}{rrrrrr} 
TABle 5. Average PAYOFFs, ASYMMETRIC GAMES (UNANIMITY RULE) \\
\hline \multicolumn{7}{c}{ Conditional on initial role assignments: } \\
$r$ & proposer & responder 1 & responder 2 & total & $\mathrm{N}$ \\
\hline$(0,20,40)$ & 12.8 & 26.6 & 44.0 & 83.3 & 130 \\
$(20,0,40)$ & 27.8 & 12.3 & 43.7 & 83.7 & 140 \\
$(40,0,20)$ & 45.1 & 12.4 & 26.5 & 84.0 & 130 \\
\hline$(0,0,60)$ & 13.0 & 12.7 & 58.7 & 84.4 & 270 \\
$(60,0,0)$ & 58.5 & 11.3 & 11.3 & 81.2 & 130 \\
\hline \multicolumn{5}{c}{ Ex ante expected payoffs } \\
$r$ & player 1 & player 2 & player 3 & total & $\mathrm{N}$ \\
\hline$(0,20,40)$ & 12.5 & 27.0 & 44.2 & 83.7 & 400 \\
$(0,0,60)$ & 12.3 & 12.3 & 58.7 & 83.4 & 400 \\
\hline
\end{tabular}

Result 3c. In asymmetric situations and under unanimity rule, ex ante expected payoffs are increasing in disagreement values.

\subsection{Asymmetric $r$ : Majority rule}

Figures 8 and 9 summarize the frequency of proposals and the passage rates under majority rule in the asymmetric conditions $(r=(0,20,40)$ and $r=(0,0,60))$. These figures are constructed analogously to Figures 4 and 5. As above, a number of patterns are immediately discernible.

The first is that most proposers attempt to build minimal winning coalitions (see Table 6). That is, most proposals are located along the axes. Pooling all proposals in the asymmetric conditions, $65 \%$ are of this type $(76 \%$ in the final 10 periods). At $52 \%$, the frequency of $\mathrm{MWC}$ is slightly lower when the proposer's $r_{i}=60$.

\footnotetext{
${ }^{32}$ Using a Wilcoxon matched-pairs signed-ranks test, we can establish that the payoff achieved by the player with the largest disagreement value is different from the payoff achieved by the other players $(p<0.001)$. (In fact, the average payoff to the player with the largest disagreement value is larger than the others within all 10 matching groups in the unanimity rule treatment.) Similarly, in the $r=(0,20,40)$ condition the average realized payoffs of the players with $r_{i}=0$ and $r_{i}=20$ are also statistically different $(p<0.01)$.
} 
Figure 8. Proposals and passage Rates, $r=(0,0,60)$, MAJORITY RULE $(\mathrm{N}=400)$

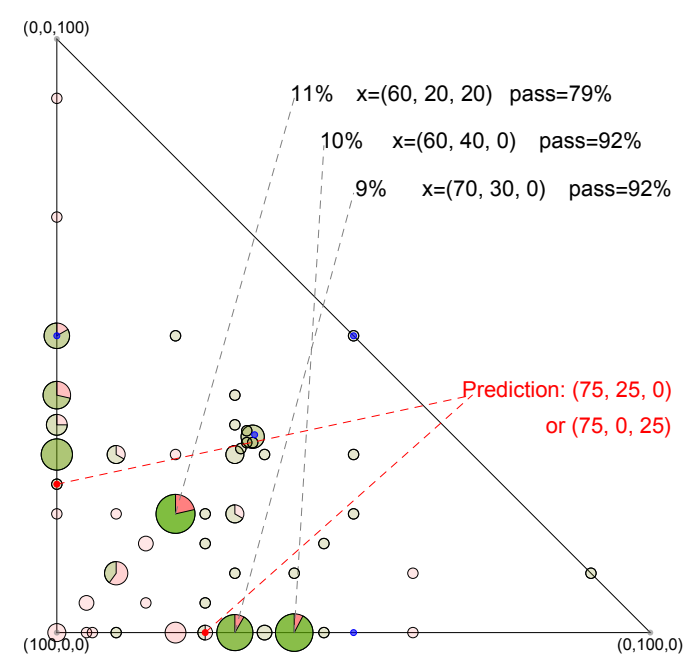

(a) $r=(60,0,0)(\mathrm{N}=130)$

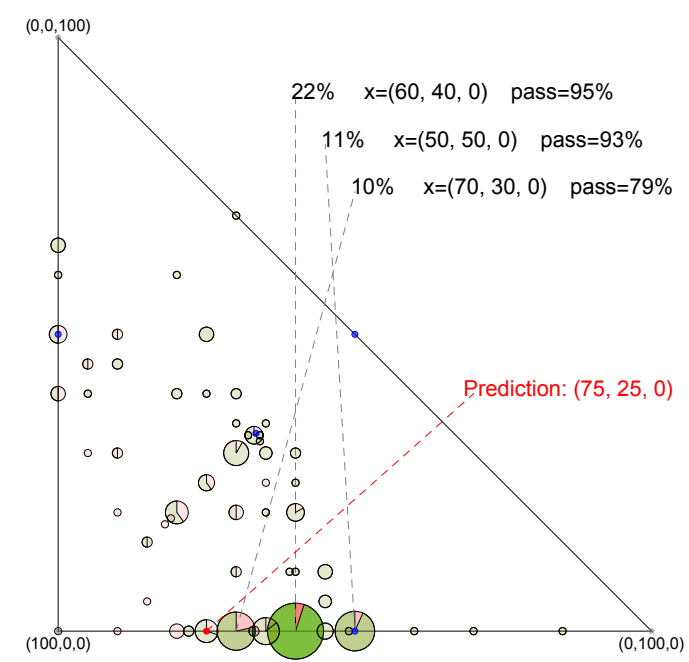

(b) $r=(0,0,60)(\mathrm{N}=270)$

Result 4. In asymmetric situations under majority rule, most proposers attempt to build minimal winning coalitions.

A second pattern emerges when we focus on situations where responder disagreement values differ (Figure 8(b) and Figure 9). In these cases, minimal winning coalitions are virtually always formed with responder 2 (with the smaller $r_{i}$ ). That is, most proposals are located along the horizontal axis. Pooling all situations where responder disagreement values differ, $57 \%$ of proposals are of this type. This is $85 \%$ of all proposed minimal winning coalitions. This number increases to $71 \%$ in the final ten periods (90\% of all minimal winning coalitions).

We test Hypothesis 4a by estimating a random-effects probit regression. We estimate the effect of disagreement values on the probability of 'being in the coalition' (receiving a positive offer), controlling for the period and the block order. ${ }^{33}$ We study this effect (Table 7 ) for the $r=(0,20,40)$ condition (left column) and the $r=(0,0,60)$ condition (right column) separately. In each case, the probability of being included is decreasing in disagreement values.

Result 4a. Under majority rule, when responder disagreement values differ, the responder with the higher disagreement value is less often included in the coalition.

\footnotetext{
${ }^{33}$ The empirical specification is similar to the voting equation described in footnote 30 .
} 
Figure 9. Proposals and passage rates, $r=(0,20,40)$, MAJORITY RULE $(\mathrm{N}=400)$

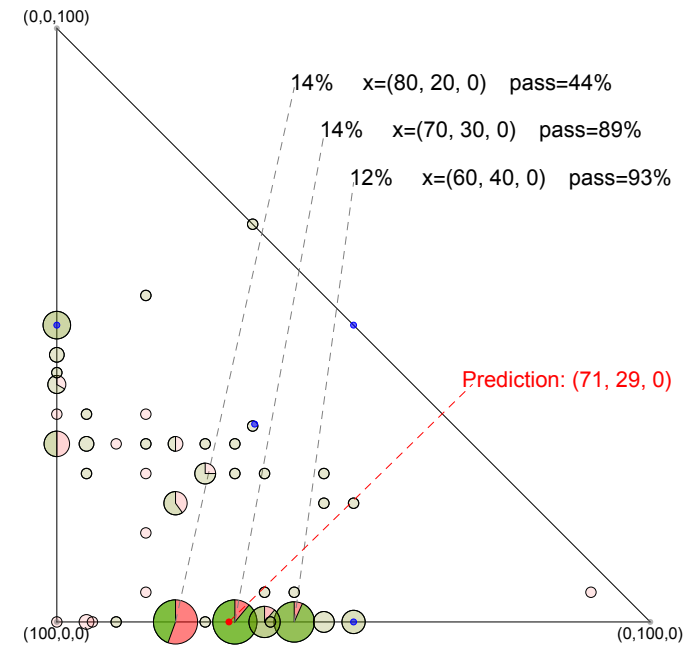

(a) $r=(40,0,20)(\mathrm{N}=130)$

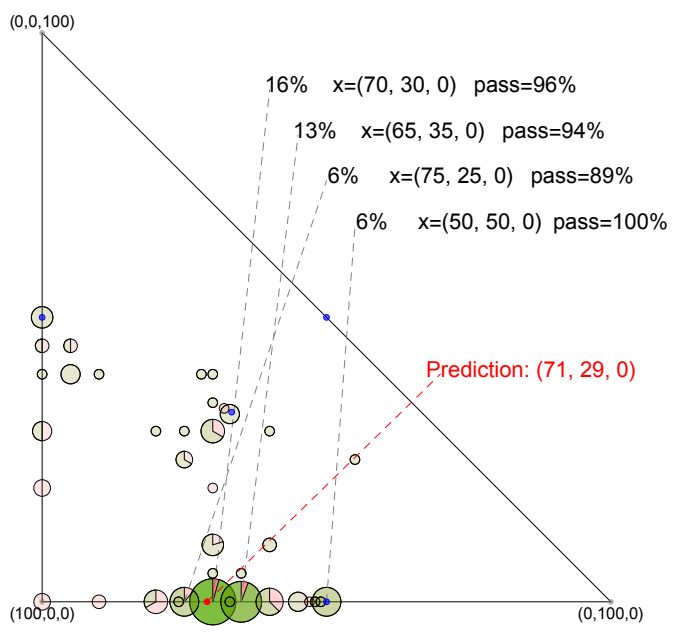

(b) $r=(20,0,40)(\mathrm{N}=140)$

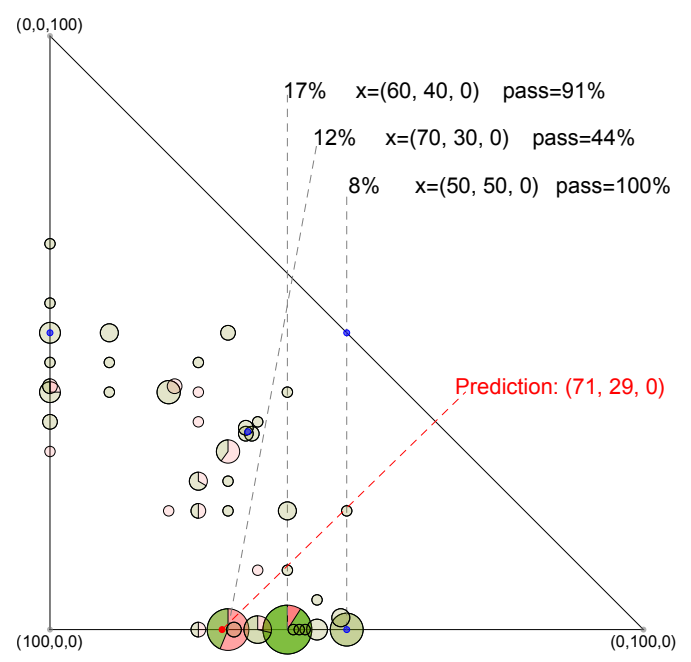

(c) $r=(0,20,40)(\mathrm{N}=130)$ 
TABle 6. Coalition COMPosition, ASymmetric Situations

MAJORITY RULE

\begin{tabular}{rrrr|rrr}
\hline & \multicolumn{3}{c}{ all periods } & \multicolumn{3}{c}{ periods 21 - 30 } \\
Situation & resp. 1 & resp. 2 & coalition & MWC with & grand \\
resp. 1 & resp. 2 & coalition \\
\hline$(0,20,40)$ & $51 \%$ & $12 \%$ & $37 \%$ & $60 \%$ & $13 \%$ & $27 \%$ \\
$(20,0,40)$ & $60 \%$ & $11 \%$ & $27 \%$ & $79 \%$ & $5 \%$ & $16 \%$ \\
$(40,0,20)$ & $58 \%$ & $15 \%$ & $26 \%$ & $69 \%$ & $10 \%$ & $21 \%$ \\
\hline$(0,0,60)$ & $59 \%$ & $6 \%$ & $35 \%$ & $73 \%$ & $6 \%$ & $20 \%$ \\
$(60,0,0)$ & $29 \%$ & $23 \%$ & $45 \%$ & $42 \%$ & $17 \%$ & $40 \%$ \\
\hline All & $53 \%$ & $12 \%$ & $34 \%$ & $66 \%$ & $9 \%$ & $24 \%$ \\
\hline
\end{tabular}

Notes: 'Situations' are defined such that the first coordinate is the proposer, the second and third responder $r_{i}$ values; minimal winning coalitions are defined as proposals that allocate a positive share to only one responder; grand coalitions are proposals that allocate positive shares to both responders.

TABle 7. REgRession ANALYsis of THE PROBABILITy OF BEING IN THE COALITION

\begin{tabular}{lcc}
\hline \hline \multicolumn{3}{c}{ Dependent variable: "Inclusion in the coalition" } \\
Participant random effects included in all models \\
\hline & Asymmetric $r$ & Asymmetric $r$ \\
& $r=(0,20,40)$ & $r=(0,0,60)$ \\
\hline Period & -0.023 & $-0.073^{* * *}$ \\
& $(0.019)$ & $(0.021)$ \\
$R r=20$ & $-0.900^{* * *}$ & \\
& $(0.147)$ & \\
$R r=40$ & $-1.662^{* * *}$ & \\
& $(0.163)$ & $-1.769^{* * *}$ \\
$R r=60$ & & $(0.172)$ \\
& & $2.863^{* * *}$ \\
Constant & $1.515^{* * *}$ & $(0.591)$ \\
& $(0.347)$ & Yes \\
Order dummies & Yes & 800 \\
Observations & 800 & 120 \\
Number of id & 120 & \\
\hline \hline
\end{tabular}

Notes: Sample includes offers to all players; dependent variable $=1$ if the player receives a positive offer, $=0$ otherwise; $R r$ is responder's $r$ value; marginal effects taken at variables means from random effect probit regressions presented; standard errors in parentheses. ${ }^{* *}$ - sig. at $1 \%{ }^{* *}$ - sig. at $5 \%{ }^{*}$ - sig. at $10 \%$.

The third pattern visible in Figures 8 and 9 is that passage rates are generally higher than under unanimity rule. Pooling all periods and asymmetric situations, $78 \%$ of proposals pass in the first round ( $82 \%$ in the final ten periods).

Figures 10 and 11 show voting behavior under majority rule in the two asymmetric conditions. The ten panels in these two figures show the same pattern: the 
responder 'included' in the coalition typically votes yes, and the excluded responder almost always votes no. To investigate the effect of disagreement values on voting, we estimate a regression model as described in footnote 30 for majority rule. Results are presented in columns 2 and 4 of Table 4 . As predicted, the coefficients on all positive $r_{i}$ values are negative and significant, suggesting that players with $r_{i}=0$ vote 'yes' more often than others. Likewise, when the responders' disagreement values differ (column 2), the coefficient on $R_{i}=40$ is larger (in absolute value) than the coefficient on $R_{i}=20 .{ }^{34}$ Although acceptance thresholds are not directly observable, these results are consistent with Hypothesis $4 \mathrm{~b}$.

Result 4b. In asymmetric situations and under majority rule, the likelihood that a player votes yes on a given offer is decreasing in her disagreement value.

Finally, we look at payoffs. Table 8 is constructed analogously to table 5 in the previous section. Focusing on ex ante expected payoffs, we only find a statistically significant disadvantage in terms of payoffs when $r_{i}=60 .^{35}$

TAble 8. Average Payoffs, asymmetric games (MAJORity RUle)

\begin{tabular}{|c|c|c|c|c|c|}
\hline \multicolumn{6}{|c|}{ Conditional on initial role assignments: } \\
\hline$r$ & proposer & responder 1 & responder 2 & total & $\mathrm{N}$ \\
\hline$\overline{(0,20,40)}$ & 44.4 & 30.7 & 20.5 & 95.6 & 130 \\
\hline$(20,0,40)$ & 49.6 & 27.0 & 18.3 & 94.9 & 140 \\
\hline$(40,0,20)$ & 52.6 & 26.1 & 16.8 & 95.5 & 130 \\
\hline$(0,0,60)$ & 46.0 & 31.5 & 18.1 & 95.6 & 270 \\
\hline$(60,0,0)$ & 49.2 & 21.9 & 21.9 & 92.9 & 130 \\
\hline \multicolumn{6}{|c|}{ Ex ante expected payoffs } \\
\hline$r$ & player 1 & player 2 & player 3 & total & $\mathrm{N}$ \\
\hline$(0,20,40)$ & 32.5 & 32.4 & 30.4 & 95.3 & 400 \\
\hline$(0,0,60)$ & 33.1 & 33.1 & 28.5 & 94.7 & 400 \\
\hline
\end{tabular}

Result 4c. In asymmetric situations and under majority rule, the player with the largest disagreement value achieves a lower average payoff. However, a statistically significant disadvantage is found only when his disagreement value is very large compared to others.

\footnotetext{
${ }^{34} \mathrm{~A}$ linear restriction test, however, suggests that the coefficients of these two variables are statistically indistinguishable $(p=0.142)$.

${ }^{35}$ Using a Wilcoxon matched-pairs signed-ranks test, we can establish that the payoff achieved by the player with the largest disagreement value is different from the payoff achieved by the other players when $r_{i}=60(p<0.05)$. If we look only at interactions that ended in agreement, we also find a statistically significant disadvantage in the $(0,20,40)$ condition.
} 
Figure 10. Voting Behavior, $r=(0,0,60)$, majority Rule $(\mathrm{N}=400)$

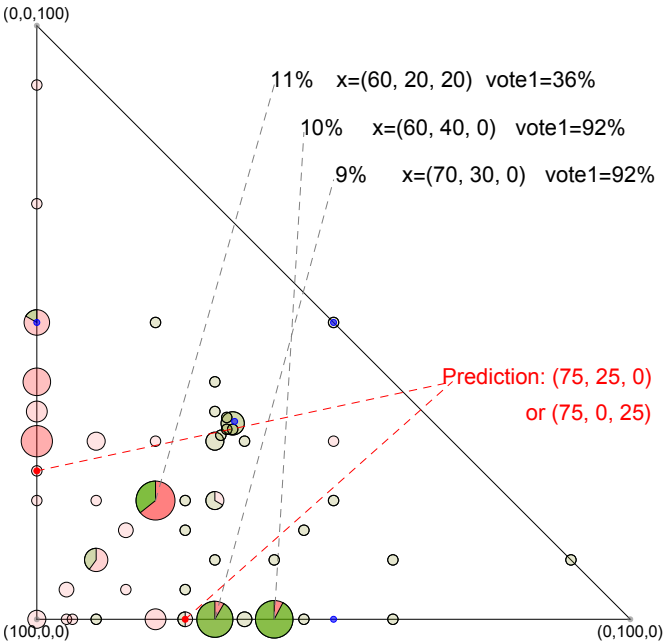

(a) $r=(60,0,0)$, Responder 1's vote

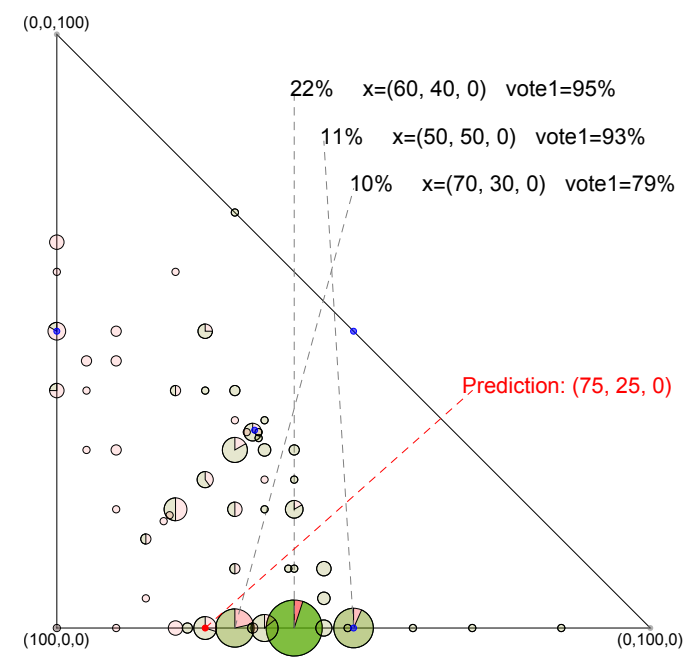

(c) $r=(0,0,60)$, Responder 1's vote

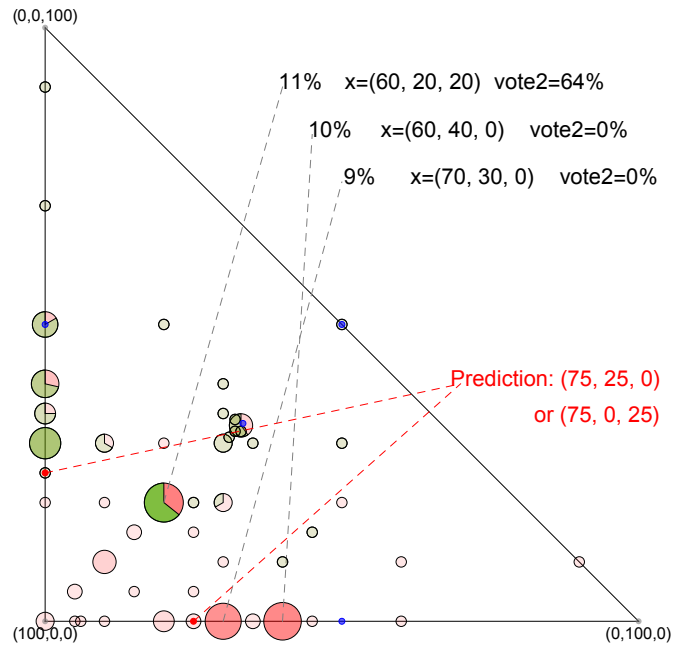

(b) $r=(60,0,0)$, Responder 2's vote

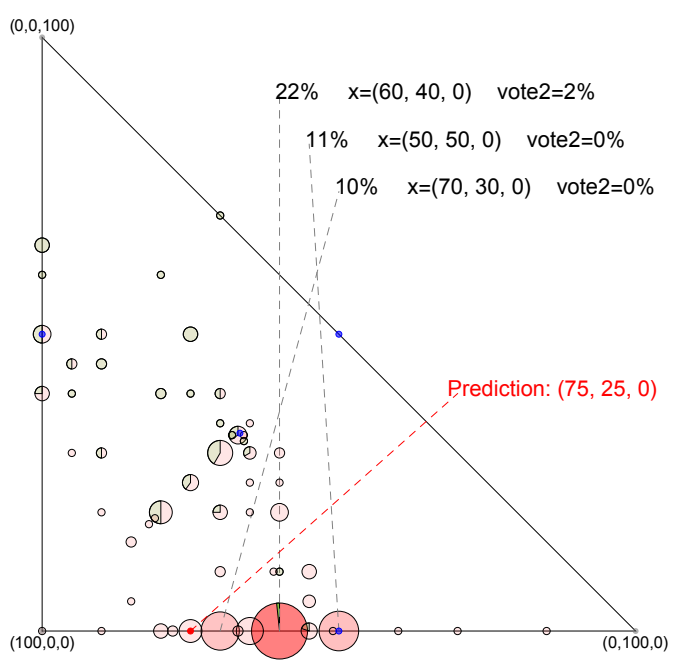

(d) $r=(0,0,60)$, Responder 2's vote 
Figure 11. Voting Behavior, $r=(0,20,40)$, majority Rule $(\mathrm{N}=400)$

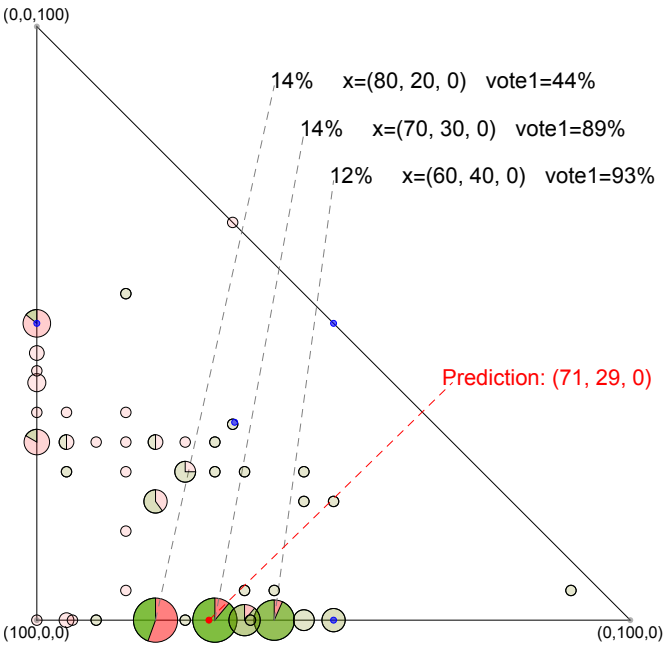

(a) $r=(40,0,20)$, Responder 1's vote

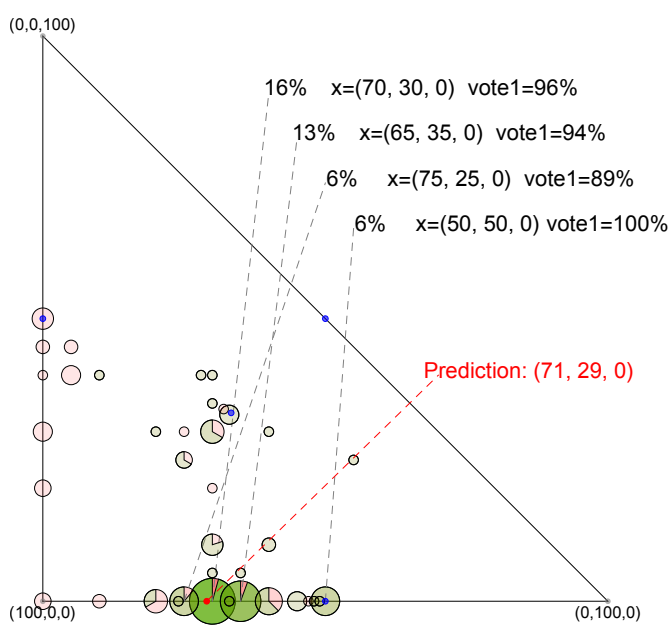

(c) $r=(20,0,40)$, Responder 1's vote

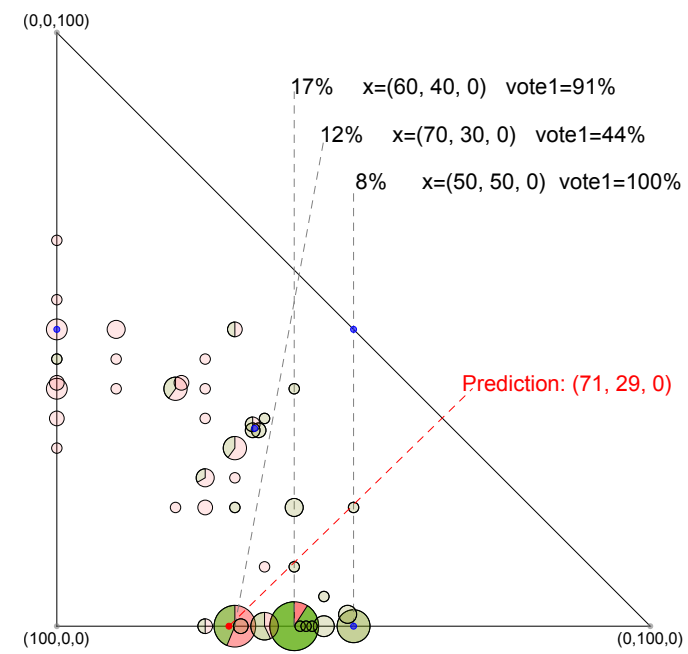

(e) $r=(0,20,40)$, Responder 1's vote

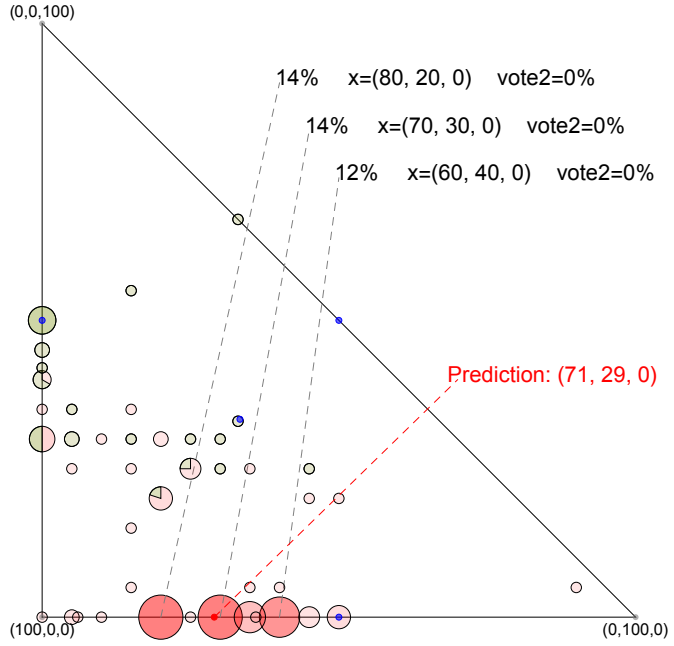

(b) $r=(40,0,20)$, Responder 2's vote

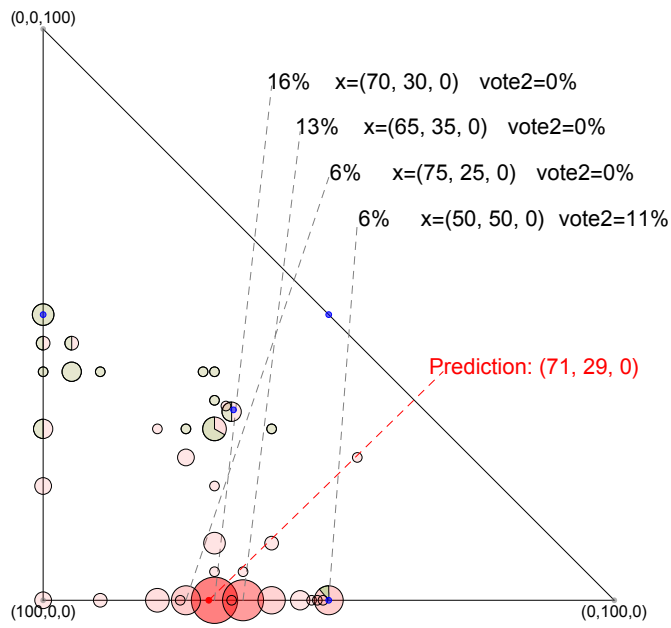

(d) $r=(20,0,40)$, Responder 2's vote

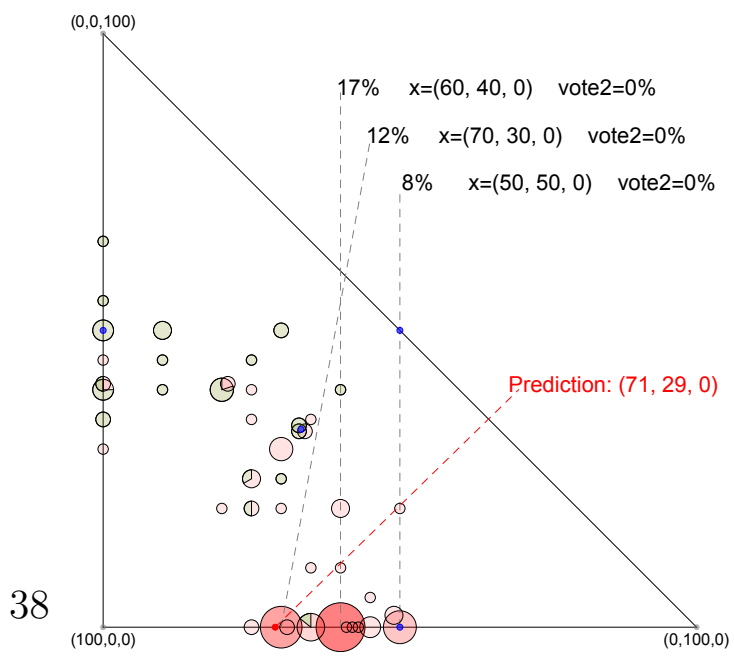

(f) $r=(0,20,40)$, Responder 2's vote 


\section{Conclusion}

We study a legislative bargaining game in which failure to agree in a given round may result in a breakdown of negotiations. In that case, each player receives an exogenous 'disagreement value'. We characterize the set of stationary subgame perfect equilibria under all q-majority rules. Under unanimity rule, equilibrium payoffs are strictly increasing in disagreement values. Under all less-than-unanimity rules, expected payoffs are either decreasing or first increasing and then decreasing in disagreement values.

We conducted an experiment designed to investigate games involving 3 players, comparing majority and unanimity rule treatments. Our results lend qualitative support for several of our main predictions. Specifically, we find that the player with the largest disagreement value indeed achieves the largest payoff under unanimity rule. Under majority rule, however, that player is included in others' coalitions significantly less often. Nonetheless, we do not find that this results in consistently lower average payoffs. A statistically significant disadvantage in terms of expected payoffs is found only if the highest disagreement value is very large compared to others. In addition, we find a large and significant difference in delay under the two decision rules, with many more first round proposals failing under unanimity rule.

Substantively, our results support the notion that 'being tough' (having a large disagreement value) may be advantageous under unanimity rule, but bad under majority rule. This, in turn, suggests that more inclusive decision rules may create incentives for players to 'act' tough, possibly leading to greater delay. In ongoing theoretical work, we are studying a version of the game in which disagreement values are privately known. Our main conjecture is that, in such a game, players may attempt to 'signal' a higher disagreement value by acting 'tough' under unanimity rule, but not under majority rule.

Acknowledgement. This research has been partly funded by Ikerbasque, the Basque Foundation of Science.

\section{References}

[1] Agranov, M. and C. Tergiman (2014) Communication in multilateral bargaining. Journal of Public Economics 118, 75-85.

[2] Agranov, M. and C. Tergiman (2016) Communication and voting rules in bargaining games. Unpublished manuscript.

[3] Anbarci, N. and N. Feltovich (2013) How sensitive are bargaining outcomes to changes in disagreement payoffs? Experimental Economics 16, 560-596. 
[4] Banks, J. S. and J. Duggan (2006) A general bargaining model of legislative policy-making. Quarterly Journal of Political Science 1, 49-85.

[5] Baranski, A. (2016) Pre-distribution: Bargaining over incentives with endogeneous production. Unpublished manuscript.

[6] Baron, D. P., and J. A. Ferejohn (1989) Bargaining in legislatures. American Political Science Review 83, 1181-1206.

[7] Battaglini, M. and T. Palfrey (2012) The dynamics of redistributive politics. Economic Theory 49, 739-777.

[8] Battaglini, M., Nunnari, S. and T. R. Palfrey (2012) Legislative bargaining and the dynamics of public investment. American Political Science Review 106, 407-429.

[9] Binmore, K. (1987) Perfect equilibria in bargaining models, in The economics of bargaining. Oxford: Blackwell, ed. by K. Binmore and P. Dasgupta.

[10] Binmore, K., Rubinstein, A. and A. Wolinsky (1986) The Nash bargaining solution in economic modelling. The RAND Journal of Economics 17, 176188.

[11] Bradfield, A. J. and J. H. Kagel (2015) Legislative bargaining with teams. Games and Economic Behavior 93, 117-127.

[12] Buchanan, J. and G. Tullock (1962) The Calculus of Consent: Logical Foundations of Constitutional Democracy. University of Michigan Press, Ann Arbor MI.

[13] Christiansen, N. (2015) Greasing the wheels: Pork and public goods contributions in a legislative bargaining experiment. Journal of Economic Behavior and Organization 120, 64-79.

[14] Christiansen, N., Georganas, S. and J. H. Kagel (2013) Coalition formation in a legislative voting game. American Economic Journal: Microeconomics 6, 182-204.

[15] Diermeier, D. and S. Gailmard (2006) Self-interest, inequality and entitlement in majoritarian decision-making. Quarterly Journal of Political Science 1, 327350.

[16] Diermeier, D. and R. Morton (2005) Proportionality versus perfectness: Experiments in majoritarian bargaining, in Social Choice and Strategic Behavior: Essays in the Honor of Jeffrey S. Banks, ed. by David Austen-Smith and John Duggan. Berlin: Springer. 
[17] Drouvelis, M., Montero, M. and M. Sefton (2010) Gaining power through enlargement: Strategic foundations and experimental evidence. Games and Economic Behavior 69, 274-292.

[18] Eraslan, H. (2002) Uniqueness of stationary equilibrium payoffs in the BaronFerejohn model. Journal of Economic Theory 103, 11-30.

[19] Eraslan, H. and McLennan, A. (2013) Uniqueness of stationary equilibrium payoffs in coalitional bargaining. Journal of Economic Theory 148, 2195-2222.

[20] Fischbacher, U. (2007) z-Tree: Zurich Toolbox for Ready-Made Economic Experiments, Experimental Economics 10, 171-178.

[21] Fréchette, G. R., Kagel, J. H. and S. F. Lehrer (2003) Bargaining in legislatures: An experimental investigation of open versus closed amendment rules. American Political Science Review 97, 221-32.

[22] Fréchette, G. R., Kagel, J. H. and M. Morelli (2005a). Nominal bargaining power, selection protocol and discounting in legislative bargaining. Journal of Public Economics 89, 1497-1517.

[23] Fréchette, G. R., Kagel, J. H. and M. Morelli (2005b). Gamson's law versus non-cooperative bargaining theory. Games and Economic Behavior 51, 365390 .

[24] Fréchette, G. R., Kagel, J. H. and M. Morelli (2005c). Behavioral identification in coalitional bargaining: An experimental analysis of demand bargaining and alternating offers. Econometrica 73, 1893-1937.

[25] Fréchette, G. R., Kagel, J. H. and M. Morelli (2012) Pork versus public goods: An experimental study of public good provision within a legislative bargaining framework. Economic Theory 49, 779-800.

[26] Harris, T. and J.W. Hardin (2013) Exact Wilcoxon signed-rank and Wilcoxon Mann-Whitney ranksum tests. Stata Journal 13(2), 337-343.

[27] Harstad, B. (2010) Strategic delegation and voting rules. Journal of Public Economics 94, 102-113.

[28] Herings, P.J.J., Meshalkin, A. and A. Predtetchinski. (2017) A one-period memory folk theorem for multilateral bargaining games. Games and Economic Behavior 103, 185-198.

[29] Jackson, M. and B. Moselle (2002) Coalition and party formation in a legislative voting game. Journal of Economic Theory 103, 49-87. 
[30] Kagel, J. H., Sung, H. and E. Winter (2010) Veto power in committees: an experimental study. Experimental Economics 13, 167-188.

[31] Kalandrakis, T. (2004) A three-player dynamic majoritarian bargaining game. Journal of Economic Theory 116, 294-322.

[32] Kalandrakis, T. (2015) Computation of equilibrium values in the Baron and Ferejohn bargaining model. Games and Economic Behavior 94, 29-38.

[33] Kawamori, T. (2005) Players' patience and equilibrium payoffs in the BaronFerejohn model. Economics Bulletin 3, 1-7.

[34] McKelvey, R.D. (1991) An experimental test of a stochastic game model of committee bargaining. In Contemporary Laboratory Research in Political Economy (ed. by Thomas R. Palfrey). Ann Arbor: University of Michigan Press.

[35] Miller, L. and C. Vanberg (2013) Decision costs in legislative bargaining: an experimental analysis. Public Choice 154, 373-394.

[36] Miller, L. and C. Vanberg (2015) Group size and decision rules in legislative bargaining. European Journal of Political Economy 37, 288-302.

[37] Miller, L., Montero, M., and C. Vanberg (2015) Legislative bargaining with heterogeneous disagreement values. Available at SSRN: https://ssrn.com/abstract $=2707386$.

[38] Montero, M. (2007) Inequity aversion may increase inequity. Economic Journal 117, C192-C204.

[39] Norman, P. (2002) Legislative bargaining and coalition formation. Journal of Economic Theory 102, 322-353.

[40] Nunnari, S. and J. Zapal (2016) Gambler's fallacy and imperfect best response in legislative bargaining. Games and Economic Behavior 99, 275-294.

[41] Ochs, J. and A. Roth (1989) An experimental study of sequential bargaining. American Economic Review 79, 355-384.

[42] Okada, A. (1996) A noncooperative coalitional bargaining game with random proposers. Games and Economic Behavior 16, 97-108.

[43] Rubinstein, A. (1982) Perfect equilibrium in a bargaining model. Econometrica 50, 97-108. 
[44] Volden, C. and A.E. Wiseman (2007) Bargaining in legislatures over particularistic and collective goods. American Political Science Review 101, 79-92.

[45] Volden, C. and A.E. Wiseman (2008) Erratum to "Bargaining in legislatures over particularistic and collective goods". American Political Science Review 102, 385-386. 


\section{A Appendix}

\section{A.1 Characterization of equilibrium}

For reasons that will become clear, it is possible and convenient to formulate a set of necessary and sufficient conditions for an SSPE in terms of the continuation values associated with the equilibrium strategies.

Lemma 1. Let $\sigma=\left(\sigma_{1}, \ldots, \sigma_{n}\right)$ be a combination of stationary strategies and $\left(z_{1}, \ldots, z_{n}\right)$ its associated vector of continuation values. The strategy combination $\sigma$ is an SSPE if and only if the following conditions are satisfied:

1. As a responder, player $i$ votes 'yes' on any proposal with $x_{i}>z_{i}$ and 'no' on any proposal with $x_{i}<z_{i}$.

2. If $\min _{S: S \ni i,|S|=q} \sum_{j \in S} z_{j}<1$, the only proposals that player $i$ makes with a positive probability as a proposer are such that $x_{j}=z_{j}$ for all $j \in T \backslash\{i\}, x_{i}=$ $1-\sum_{j \in T \backslash\{i\}} z_{j}$ and $x_{j}=0$ for all $j \in N \backslash T$, where $T \in \arg \min _{S: S \ni i,|S|=q} \sum_{j \in S} z_{j}$. These proposals are always accepted.

3. If $\min _{S: S \ni i,|S|=q} \sum_{j \in S} z_{j}>1$, player $i$ always makes a proposal that would be rejected.

Proof. 1. This follows from subgame perfection and our assumption that players always vote as if they are pivotal.

2. Suppose $\min _{S: S \ni i,|S|=q} \sum_{j \in S} z_{j}<1$ and let $T \in \arg \min _{S \ni i,|S|=q} \sum_{j \in S} z_{j}$. Player $i$ can propose $x_{j}=z_{j}+\epsilon$ for $j \in T \backslash\{i\}, x_{j}=0$ for $j \in N \backslash T$ and $x_{i}=1-$ $\sum_{j \in T \backslash\{i\}} z_{j}-(q-1) \epsilon$ for a sufficiently small $\epsilon>0$. This proposal would be accepted and gives player $i$ a payoff above $z_{i}$, which would be the payoff from making a proposal that would be rejected. Hence, player $i$ will never make a proposal that would be rejected since there is a more favorable proposal that would be accepted. Take any of the proposals that player $i$ makes with positive probability in equilibrium, and let $Q$ be the set that votes in favor of this proposal. It must be the case that $x_{j}=z_{j}$ for all $j \in Q \backslash\{i\}$, since $x_{j}<z_{j}$ would lead to a rejection and $x_{j}>z_{j}$ could not be optimal since player $i$ could always do better by reducing $x_{j}$ while keeping the inequality $x_{j}>z_{j}$. Also, $Q \in \arg \min _{S: S \ni i,|S|=q} \sum_{j \in S} z_{j}$, since otherwise player $i$ could do better by proposing coalition $T$ and offering $z_{j}+\epsilon$ to each player in $T \backslash\{i\}$ for a sufficiently small $\epsilon$.

3. If $\min _{S: S \ni i,|S|=q} \sum_{j \in S} z_{j}>1$, it is not possible to find a proposal that would give player $i$ and $q-1$ other players at least their continuation value, hence it is optimal for player $i$ to make a proposal that will be rejected.

Note that the concept of SSPE imposes no restrictions on behavior in the knife-edge case $\min _{S: S \ni i,|S|=q} \sum_{j \in S} z_{j}=1$. Player $i$ may or may not offer their continuation value to players in some $T \in \arg \min _{S: S \ni i,|S|=q} \sum_{j \in S} z_{j}$ and, even if 
player $i$ does, the remaining players in $T$ may or may not accept the proposal. However, such a situation can only arise if disagreement values are very high. As we will see in section A.3 (lemma 3 and corollary 1), $\sum_{j=1}^{q-1} r_{j}+r_{n}<1$ implies that $\min _{S: S \ni i,|S|=q} \sum_{j \in S} z_{j}<1$ for all $i$.

Note also that the lemma does not constrain players to vote yes to all proposals with $x_{i}=z_{i}$. In principle they could vote no, but only in the aforementioned case $\arg \min _{S: S \ni i,|S|=q} \sum_{j \in S} z_{j}=1$ or off the equilibrium path, i.e, as a response to a proposal that is never actually made.

In our discussion henceforth we will assume that both proposers and responders break ties in favor of agreement. ${ }^{36}$

\section{A.2 Ranking of continuation values}

The characterization of equilibrium above depends on the continuation values $z_{i}$; these values are endogenous. It will be useful to have results in terms of the exogenous disagreement values $r_{i}$. The following lemma shows that the $z_{i}$ values are ranked in the same order as the $r_{i}$ values, though as we will see some strict inequalities may become weak inequalities.

Lemma 2. Let $r_{i} \geq r_{j}$. Then $z_{i} \geq z_{j}$ in any $S S P E$.

Proof. Suppose $r_{i} \geq r_{j}$ but $z_{j}>z_{i}$.

If $\min _{S: S \ni j,|S|=q} \sum_{k \in S} z_{k}>1$, player $j$ never makes acceptable proposals. In this case nobody would make acceptable proposals involving player $j$ and it is clear that $z_{j} \leq z_{i}$, since $i$ gets at least the same as $j$ in the event of disagreement and may get something in the event of agreement (while $j$ is sure to get nothing).

Now consider the case $\min _{S: S \ni j,|S|=q} \sum_{k \in S} z_{k} \leq 1$, so that player $j$ finds it profitable to make acceptable proposals (and so does player $i$ ). Delay is not completely ruled out in this case, since there may be players other than $i$ and $j$ who cannot find a profitable coalition. Denote by $\alpha$ the probability that one of those players is selected to make a proposal; $\alpha \geq 0 .{ }^{37}$

Denoting player $k$ 's payoff conditional on being proposer as $\pi_{k}$, continuation

\footnotetext{
${ }^{36}$ Eraslan and McLennan (2013, appendix A) formally show that there is little loss of generality in requiring responders to accept proposals when indifferent. Banks and Duggan (2006) incorporate this tie-breaking rule into their definition of equilibrium.

${ }^{37}$ This paragraph assumes that ties are always broken in favor of agreement. If we do not impose this, player $j$ could make proposals that are rejected with positive probability if $\min _{S: S \ni j,|S|=q} \sum_{k \in S} z_{k}=1$. This does not affect the discussion on $\pi_{i}$ and $\pi_{j}$. We would then have two separate values, $\alpha_{i}$ and $\alpha_{j}$, where $\alpha_{k}$ would be the probability that a proposal is made by a player other than $k$ and rejected. We would then have $\alpha_{i} \geq \alpha_{j}$, which goes in the same direction as $\mu_{i} \geq \mu_{j}$, and the proof can be easily adapted.
} 
values are given by

$$
\begin{aligned}
& z_{i}=\frac{\delta}{n} \pi_{i}+\delta\left[\mu_{i}-\frac{1}{n}+\alpha\right] z_{i}+(1-\delta) r_{i} \\
& z_{j}=\frac{\delta}{n} \pi_{j}+\delta\left[\mu_{j}-\frac{1}{n}+\alpha\right] z_{j}+(1-\delta) r_{j}
\end{aligned}
$$

The inequality $z_{j}>z_{i}$ implies $\pi_{i} \leq \pi_{j}$, but the difference $\pi_{j}-\pi_{i}$ cannot exceed $z_{j}-z_{i}$. We also know that $\mu_{i} \geq \mu_{j}$. This is because players other than $i$ and $j$ would never include $j$ in the coalition and exclude $i$. As for $i$ and $j$ themselves, suppose player $j$ does not propose to player $i$ with certainty. Then there must be a coalition $T$ such that $j \in T, i \notin T$ that is optimal for player $j$, that is, $\sum_{k \in T} z_{k} \leq \sum_{k \in S} z_{k}$ for all $S \supseteq\{i, j\}$. But then $\sum_{k \in T \backslash\{j\} \cup\{i\}} z_{k}<\sum_{k \in S} z_{k}$ for all $S \supseteq\{i, j\}$, that is, player $i$ would never involve player $j$ in the coalition as $T \backslash\{i\} \cup\{j\}$ would be strictly cheaper than any coalition involving $j$.

$$
\begin{aligned}
z_{i} & =\frac{\delta}{n} \pi_{i}+\delta\left[\mu_{j}+\left(\mu_{i}-\mu_{j}\right)-\frac{1}{n}+\alpha\right] z_{i}+(1-\delta) r_{i} \\
z_{j} & \leq \frac{\delta}{n}\left(\pi_{i}+z_{j}-z_{i}\right)+\delta\left[\mu_{j}-\frac{1}{n}+\alpha\right] z_{j}+(1-\delta) r_{j}
\end{aligned}
$$

Subtracting and collecting terms

$$
\begin{aligned}
z_{j}-z_{i} & \leq \frac{\delta}{n}\left(z_{j}-z_{i}\right)+\delta\left[\mu_{j}-\frac{1}{n}+\alpha\right]\left(z_{j}-z_{i}\right)-\left(\mu_{i}-\mu_{j}\right) z_{i}-(1-\delta)\left(r_{i}-r_{j}\right) \\
\left(z_{j}-z_{i}\right)\left(1-\delta \mu_{j}-\delta \alpha\right) & \leq-\left(\mu_{i}-\mu_{j}\right) z_{i}-(1-\delta)\left(r_{i}-r_{j}\right)
\end{aligned}
$$

Since the LHS is strictly positive and the RHS is nonpositive, we have a contradiction.

\section{A.3 Equilibria with immediate agreement}

It will be useful to distinguish between equilibria in which all players make acceptable proposals and equilibria in which some players do not. We will refer to the former as equilibria with no delay or immediate agreement, and to the latter as equilibria involving delay. We begin by presenting sufficient conditions for immediate agreement.

Lemma 3. If there exists a coalition $S$ with $|S|=q$ and $\sum_{j \in S} r_{j}<1$, then all players in $S$ make acceptable proposals in any SSPE.

Proof. Note that for any $S \subseteq N, \sum_{j \in S} r_{j}<1$ implies $\sum_{j \in S} z_{j}<1$ : Since total payoffs for $S$ add up to less than 1 in the event of disagreement and can add up to at most 1 in the event of agreement, the maximum possible value of $\sum_{j \in S} z_{j}$ is $\delta+(1-\delta) \sum_{j \in S} r_{j}<1$. The result then follows from part 2 of lemma 1. 
Corollary 1. If $\sum_{j=1}^{q-1} r_{j}+r_{n}<1$, any SSPE exhibits immediate agreement.

If agreement is efficient, corollary 1 implies that immediate agreement will occur in any SSPE for any decision rule. When $q<n$, corollary 1 also implies that immediate agreement may occur even if it is not efficient. In fact, the following example shows that immediate agreement may occur even in the extreme case where $\sum_{j \in S} r_{j}>1$ for all $S$ such that $|S|=q$.

Let $n=3, q=2, r_{i}=\frac{9}{15}$ and $\delta=0.5$. There is an equilibrium in which proposers offer $\frac{7}{15}$ to a randomly selected partner, and responders accept all proposals that give them at least $\frac{7}{15}$. (There is also an equilibrium in which agreement is never reached).

To see that this is an equilibrium, note that the continuation values that follow from the strategies are determined as follows. When proposing, a player receives $1-\frac{7}{15}=\frac{8}{15}$. If not proposing, they are included in the coalition with probability $\frac{1}{2}$; in this case they earn $\frac{7}{15}$. Thus, a player's expected payoff is $\frac{1}{3} \frac{8}{15}+\frac{2}{3} \frac{1}{2} \frac{7}{15}=\frac{5}{15}$. Therefore, the continuation value is $\delta \frac{5}{15}+(1-\delta) \frac{9}{15}$; since $\delta=0.5$ this is $\frac{7}{15}$.

As the above example shows, there is a very strong pressure for immediate agreement under less-than-unanimity rule. We now characterize the properties of equilibria which exhibit immediate agreement. It will be useful to define the following sets:

$$
\begin{aligned}
\mathcal{L} & =\left\{i \in N: z_{i}<z_{q}\right\} \\
\mathcal{M} & =\left\{i \in N: z_{i}=z_{q}\right\} \\
\mathcal{H} & =\left\{i \in N: z_{i}>z_{q}\right\}
\end{aligned}
$$

That is, the set $\mathcal{L}$ is the set of players whose votes are "cheaper" than that of player $q$, and the set $\mathcal{M}$ contains all players who are as expensive as player $q$, while the set $\mathcal{H}$ contains those that are strictly more expensive. Therefore, when any player proposes, an optimal strategy involves buying all players in $\mathcal{L}$ and as many players in $\mathcal{M}$ as are necessary to build a coalition of size $q$. Clearly the set $\mathcal{M}$ is always nonempty, though one or both of the sets $\mathcal{L}$ and $\mathcal{H}$ may be empty.

By lemma 2, any SSPE must have $z_{1} \leq z_{2} \leq \ldots \leq z_{n}$. Hence, in order to know the partition into the three sets it is sufficient to know their cardinalities. We will denote those cardinalities by $L, M$ and $H$ respectively.

We begin by showing that a no-delay equilibrium is characterized by $L, M$ and $H$ in the sense that the continuation values $z_{i}$, expected payoffs $y_{i}$ and inclusion probabilities $\mu_{i}$ are uniquely determined by $L, M$ and $H$, though there may be several strategy combinations that lead to the same values of $z_{i}, y_{i}$ and $\mu_{i} .{ }^{38,39}$

\footnotetext{
${ }^{38}$ We are not (yet) claiming that the no-delay equilibrium is unique, just that other no-delay equilibria would lead to different values of $L, M$ and $H$.

${ }^{39}$ It is a known feature of legislative bargaining models that a given vector of equilibrium
} 
Proposition 3. Given an SSPE with immediate agreement, the partition of the player set into the sets $\mathcal{L}, \mathcal{M}$ and $\mathcal{H}$ uniquely determines the equilibrium values of $y, z$ and $\mu$.

Proof. In a no-delay equilibrium, the proposer offers $z_{i}$ to $q-1$ other players (the ones with the $q-1$ lowest values of $\left.z_{i}\right)$ and 0 to the remaining players. ${ }^{40}$

Define $Z_{q}:=\sum_{i \leq q} z_{i}$ and $Z_{L}:=\sum_{i \in L} z_{i}$. By definition, $Z_{q}=Z_{L}+(q-L) z_{q}$. Continuation values are given by the following equations:

$$
\begin{aligned}
\mathcal{L} & : \quad z_{i}=\delta\left[\frac{1}{n}\left(1-Z_{q}+z_{i}\right)+\left(\mu_{i}-\frac{1}{n}\right) z_{i}\right]+(1-\delta) r_{i} . \\
\mathcal{M}: & z_{i}=\delta\left[\frac{1}{n}\left(1-Z_{q}+z_{i}\right)+\left(\mu_{i}-\frac{1}{n}\right) z_{i}\right]+(1-\delta) r_{i} . \\
\mathcal{H}: & : z_{i}=\delta\left[\frac{1}{n}\left(1-Z_{q}+z_{q}\right)+\left(\mu_{i}-\frac{1}{n}\right) z_{i}\right]+(1-\delta) r_{i} .
\end{aligned}
$$

As a proposer, a player buys the votes from the cheapest $q-1$ other players. Recall that the total continuation value of the cheapest $q$ players is $Z_{q}$. Players in $\mathcal{L}$ are themselves among the cheapest $q$, so they pay $Z_{q}-z_{i}$. Players in $\mathcal{H}$ are not among the cheapest $q$, so they can buy the cheapest $q-1$ votes and pay $Z_{q}-z_{q}$. Players in $\mathcal{M}$ can be thought of as paying $Z_{q}-z_{i}$ or $Z_{q}-z_{q}$, since $z_{i}=z_{q}$. Expected equilibrium payoffs are thus $y_{i}=\frac{1}{n}\left(1-Z_{q}+\min \left(z_{i}, z_{q}\right)\right)+\left(\mu_{i}-\frac{1}{n}\right) z_{i}$. Continuation values are given by $z_{i}=\delta y_{i}+(1-\delta) r_{i}$.

Consider players in $\mathcal{M}$. Collecting terms and taking into account that by definition $z_{i}=z_{q}$ in this set we find

$$
\mathcal{M}: z_{q}=\delta\left[\frac{1}{n}\left(1-Z_{q}\right)+\mu_{i} z_{q}\right]+(1-\delta) r_{i}
$$

This has a very clear interpretation: if bargaining goes on after a rejection, player $i$ gets his continuation value whenever he is part of a coalition, and on top of that he gets the proposer surplus with probability $\frac{1}{n}$ (the proposer surplus is the difference between $i$ 's payoff as a proposer and $i$ 's payoff as a coalition partner; its value for players in $\mathcal{L}$ or $\mathcal{M}$ is $1-Z_{q}$ ).

expected payoffs may be supported by several strategy combinations (see e.g. Eraslan and McLennan, 2013).

${ }^{40}$ Note that it cannot be optimal for the proposer to offer their continuation value to more than $q-1$ players because he would be better off by excluding the coalition partner with the highest $z_{i}$. The only possible exception would be if $z_{i}=0$ for more than $q-1$ players, but this is not possible since each of those players could form a coalition with the rest and get a positive payoff as a proposer, which would contradict $z_{i}=0$. 
Collecting terms again

$$
\mathcal{M}:\left(1-\delta \mu_{i}\right) z_{q}=\frac{\delta}{n}\left(1-Z_{q}\right)+(1-\delta) r_{i}
$$

We also know that $\sum_{i \in N} \mu_{i}=q$, that is, the coalition that forms always contains exactly $q$ players. Moreover, $\mu_{i}=1$ for all $i$ in $\mathcal{L}$ and $\mu_{i}=\frac{1}{n}$ for all $i$ in $\mathcal{H}$. That is, players in $\mathcal{L}$ are always included in the coalition, and players in $\mathcal{H}$ are only included when they are proposers. Hence, $\sum_{i \in M} \mu_{i}=q-L-\frac{H}{n}$. Note that $\sum_{i \in M} \mu_{i}>\frac{M}{n}$, which, since each player in $\mathcal{M}$ is the proposer with probability $\frac{1}{n}$, implies that, collectively, players in $\mathcal{M}$ have a positive probability of being coalition partners. ${ }^{41}$

If we add up all the equations (3),

$$
M z_{q}-\delta\left(q-L-\frac{H}{n}\right) z_{q}=M \frac{\delta}{n}\left(1-Z_{q}\right)+(1-\delta) \sum_{i \in M} r_{i}
$$

Dividing everything by $M$,

$$
z_{q}-\delta \frac{\left(q-L-\frac{H}{n}\right)}{M} z_{q}=\frac{\delta}{n}\left(1-Z_{q}\right)+(1-\delta) \bar{r}_{M}
$$

where $\bar{r}_{M}$ is the average value of $r$ in the set $\mathcal{M}, \frac{\sum_{i \in M} r_{i}}{M}$.

We also know $Z_{q}=Z_{L}+(q-L) z_{q}$, so we can get an equation with two unknowns, $Z_{L}$ and $z_{q}$.

$$
z_{q}-\delta \frac{\left(q-L-\frac{H}{n}\right)}{M} z_{q}=\frac{\delta}{n}\left(1-Z_{L}-(q-L) z_{q}\right)+(1-\delta) \bar{r}_{M}
$$

Collecting terms

$$
\left[1-\delta(q-L)\left(\frac{1}{M}-\frac{1}{n}\right)+\delta \frac{H}{M n}\right] z_{q}=\frac{\delta}{n}\left(1-Z_{L}\right)+(1-\delta) \bar{r}_{M}
$$

This gives us an equation where, given $H, M$ and $L$, the only unknowns are $z_{q}$ and $Z_{L}$.

If all players are in $\mathcal{M}$, the equation simplifies to $z_{q}=\frac{\delta}{n}+(1-\delta) \bar{r}_{N}$, which is clear since, assuming immediate agreement, $\sum_{i \in N} z_{i}=\delta+(1-\delta) \sum_{i \in N} r_{i}$. If all players are in $\mathcal{M}$ they all have the same continuation value, which must then equal the previous expression divided by $n$.

\footnotetext{
${ }^{41}$ If $L=0$ we have $\sum_{i \in M} \mu_{i}=q-\frac{H}{n}$, which, since $q>1$ and $H<n$ implies $\sum_{i \in M} \mu_{i}>1$. If $L>0$, by definition we have $q-L \geq 1$ and $n-H>M$, hence $\sum_{i \in M} \mu_{i} \geq 1-\frac{H}{n}=\frac{n-H}{n}>\frac{M}{n}$.
} 
For players in $\mathcal{L}$, since $\mu_{i}=1$, we find

$$
\begin{gathered}
\mathcal{L}: z_{i}=\delta\left[\frac{1}{n}\left(1-Z_{q}+z_{i}\right)+\frac{n-1}{n} z_{i}\right]+(1-\delta) r_{i} \\
z_{i}=\delta\left[\frac{1}{n}\left(1-Z_{q}\right)+z_{i}\right]+(1-\delta) r_{i} \\
z_{i}-r_{i}=\frac{\delta}{1-\delta} \frac{1}{n}\left(1-Z_{q}\right)
\end{gathered}
$$

We then see that $z_{i}-r_{i}$ is a constant, that is, all players in $\mathcal{L}$ get the same surplus above $r_{i}$.

Replacing $Z_{q}$ by its value we find

$$
z_{i}-r_{i}=\frac{\delta}{1-\delta} \frac{1}{n}\left(1-Z_{L}-(q-L) z_{q}\right)
$$

If we instead add up the equations (5) for players in $\mathcal{L}$, we find

$$
Z_{L}=\delta\left[\frac{L}{n}\left(1-Z_{q}\right)+Z_{L}\right]+(1-\delta) L \bar{r}_{L}
$$

Replacing $Z_{q}$ by its value in terms of $z_{q}$ and $Z_{L}$ and collecting terms:

$$
\left(1-\delta+\delta \frac{L}{n}\right) Z_{L}=\delta \frac{L}{n}\left(1-(q-L) z_{q}\right)+(1-\delta) L \bar{r}_{L}
$$

The set $\mathcal{H}$ is residual. For players in this set,

$$
\mathcal{H}: z_{i}=\frac{\delta}{n}\left(1-Z_{q}+z_{q}\right)+(1-\delta) r_{i}
$$

Clearly, $z_{i}$ is increasing in $r_{i}$ for players in the set $\mathcal{H}$.

The values $Z_{L}$ and $z_{q}$ can be found from the system of two linear equations and two unknowns (4) and (8). Once these two values have been found, $z_{i}$ values for players in $\mathcal{L}$ can be found from $(7)$ and $z_{i}$ values for players in $\mathcal{H}$ can be found from (9). The probabilities of inclusion in the coalition for players in $\mathcal{H}$ and $\mathcal{L}$ are known; the probabilities of inclusion $\mu_{i}$ for players in $\mathcal{M}$ can be found from (3). Expected payoffs $(y)_{i \in N}$ are found from the equation $z_{i}=\delta y_{i}+(1-\delta) r_{i}$.

Proposition 4. For $q<n$, expected equilibrium payoffs in any no-delay SSPE are strictly increasing in $r_{i}$ within the set $\mathcal{L}$, strictly decreasing in $r_{i}$ within the set $\mathcal{M}$, and constant within the set $\mathcal{H}$. Furthermore, expected payoffs for all players in $\mathcal{M}$ are at least as high as those for players in $\mathcal{H}$. 
Proof. Since players in $\mathcal{L}$ have $\mu_{i}=1$, expected payoffs for a player in $\mathcal{L}$ are given by $y_{i}=\frac{1}{n}\left(1-Z_{q}+z_{i}\right)+\frac{n-1}{n} z_{i}$. Using $(6), y_{i}=\frac{1}{n} \frac{1}{1-\delta}\left(1-Z_{q}\right)+r_{i}$. Hence, $y_{i}$ is increasing in $r_{i}$ within this set.

Players in $\mathcal{M}$ all get $1-Z_{q}+z_{q}$ as proposers and $z_{q}$ as responders, thus any difference in expected payoffs must be due to differences in $\mu_{i}$. Since $\mu_{i}$ is decreasing in $r_{i}$ by (3), $y_{i}$ is decreasing in $r_{i}$ as well.

Finally, since no player in $\mathcal{H}$ receives proposals from other players, all players in $\mathcal{H}$ have $y_{i}=\frac{1}{n}\left(1-Z_{q}+z_{q}\right)$, which does not depend on $r_{i}$.

Since players in $\mathcal{M}$ and $\mathcal{H}$ get the same payoff as proposers and players in $\mathcal{H}$ never get any proposals from other players, it is clear that expected payoffs for a player in $\mathcal{M}$ cannot be lower than those of a player in $\mathcal{H}$. Indeed, they will typically be strictly higher, except for non-generic cases in which player $L+M$ (the most expensive player in $\mathcal{M}$ ) does not get any proposals. We know that collectively the players in $\mathcal{M}$ have a positive probability of being coalition partners, hence player $L+1$ must have a strictly higher payoff than players in $\mathcal{H}$.

The sets $\mathcal{L}, \mathcal{M}$ and $\mathcal{H}$ are endogenous. Putting proposition 4 and lemma 2 together allows us to state a result in terms of the exogenous values $r_{i}$. Under all less-than-unanimity rules, expected equilibrium payoffs are either decreasing or first increasing and then decreasing in $r_{i}$. The player with the highest expected equilibrium payoff is either player $L$ or player $L+1$.

Corollary 2. The highest expected equilibrium payoff is achieved by (one or more) player(s) for whom $r_{i} \leq r_{q}$.

We can also establish how the surplus $y_{i}-r_{i}$ varies with $r_{i}$. While this surplus is shared equally under unanimity, players with the lowest disagreement values capture the greatest surplus under majority. We have seen in the proof of proposition 4 that, for players in $\mathcal{L}, y_{i}=\frac{1}{n} \frac{1}{1-\delta}\left(1-Z_{q}\right)+r_{i}$, hence all players in $\mathcal{L}$ have the same $y_{i}-r_{i}$. We also know from proposition 4 that $y_{i}$ is decreasing within $\mathcal{M}$ and constant within $\mathcal{H}$, and that $y_{L+M} \geq y_{L+M+1}$; it follows that $y_{i}-r_{i}$ is decreasing in $r_{i}$ within the set $\mathcal{M} \cup \mathcal{H}$.

Furthermore, while the highest expected equilibrium payoff $y_{i}$ may be achieved by either player $L$ or player $L+1$, the highest expected surplus is always achieved by the players in $\mathcal{L}$. In order to see this, note that, for a player in $\mathcal{M}, z_{i}=$ $\delta\left[\frac{1}{n}\left(1-Z_{q}+z_{i}\right)+\left(\mu_{i}-\frac{1}{n}\right) z_{i}\right]+(1-\delta) r_{i}$. This expression can be rewritten as $z_{i}-r_{i}=\frac{\delta}{1-\delta} \frac{1}{n}\left(1-Z_{q}\right)-\frac{\delta}{1-\delta}\left(1-\mu_{i}\right) z_{i}$. Comparing this expression with (6) we see that, since $\mu_{i} \leq 1$ and $z_{i}=z_{q}>0$ for $i$ in $\mathcal{M}$, players in $\mathcal{M}$ have a lower $z_{i}-r_{i}$ (strictly lower, except for nongeneric cases where $\mu_{i}=1$ for the players with the lowest $r_{i}$ in $\mathcal{M}$ ) and hence achieve a lower surplus as coalition partners. As proposers, players in both sets get $1-Z_{q}+z_{i}$, hence the payoff a proposer gets over and above the disagreement value, $1-Z_{q}+z_{i}-r_{i}$, is higher for players in 
$\mathcal{L}$ since $z_{i}-r_{i}$ is greater. Given that players in $\mathcal{L}$ achieve a greater surplus both as proposers and as coalition partners (and are more likely to achieve this surplus since they have $\mu_{i}=1$ ), they have a greater surplus overall.

Corollary 3. The highest expected surplus $y_{i}-r_{i}$ is achieved by the players in $\mathcal{L}$. For players in $\mathcal{M} \cup \mathcal{H}, y_{i}-r_{i}$ is decreasing in $r_{i}$.

There may be multiple no-delay SSPE. However, all no-delay SSPE are equivalent in the sense that they lead to the same expected equilibrium payoffs, continuation values and probabilities of being included in the final coalition, though they may differ in the exact mixed strategies used, as in the original model (see Eraslan and McLennan (2013)).

Proposition 5. Let $\sigma$ and $\sigma^{\prime}$ be two no-delay SSPE. Then $y=y^{\prime}, z=z^{\prime}$ and $\mu=\mu^{\prime}$.

Proof. By contradiction, suppose $\sigma$ and $\sigma^{\prime}$ are two SSPE that induce different partitions of the set $N$. We will denote by $\mathcal{L}, \mathcal{M}$ and $\mathcal{H}$ the sets associated to $\sigma$ (the sets associated to $\sigma^{\prime}$ will be denoted by $\mathcal{L}^{\prime}, \mathcal{M}^{\prime}$ and $\mathcal{H}^{\prime}$ ). Recall that continuation values must respect the order of the disagreement values, hence if $\mathcal{L}$ and $\mathcal{L}^{\prime}$ have the same cardinality the actual sets must also coincide. Similarly, if $\mathcal{L} \neq \mathcal{L}^{\prime}$, one of the two sets must be a strict subset of the other.

Case 1. Suppose $\mathcal{L}=\mathcal{L}^{\prime}$. Then $\mathcal{M} \neq \mathcal{M}^{\prime}$ (otherwise the partitions would be identical).

1. a) Suppose $Z_{q}=Z_{q}^{\prime}$. Recall that the equation for the continuation value of a player in set $\mathcal{L}$ is

$$
z_{i}=r_{i}+\frac{\delta}{1-\delta} \frac{1}{n}\left(1-Z_{q}\right)
$$

The same equation holds in equilibrium $\sigma^{\prime}$, with $z_{i}$ being replaced by $z_{i}^{\prime}$ and $Z_{q}$ being replaced by $Z_{q}^{\prime}$.

Since by assumption $Z_{q}=Z_{q}^{\prime}$, it follows that $z_{i}=z_{i}^{\prime}$ for all players in $\mathcal{L} \cap \mathcal{L}^{\prime}$. Since, also by assumption, $\mathcal{L}=\mathcal{L}^{\prime}$, we have $Z_{L}=Z_{L^{\prime}}^{\prime}$, and (given that $Z_{q}=Z_{L}+(q-L) z_{q}$ and $\left.Z_{q}^{\prime}=Z_{L^{\prime}}^{\prime}+\left(q-L^{\prime}\right) z_{q}^{\prime}\right) z_{q}=z_{q}^{\prime}$.

Since the partitions are different, $\mathcal{M} \neq \mathcal{M}^{\prime}$. Given our result on how the $z_{i}$ values are ranked in the same way as the $r_{i}$ values, and given that $\mathcal{L}=\mathcal{L}^{\prime}$ by assumption, one of the two sets must be a subset of the other. Without loss of generality, let $\mathcal{M} \varsubsetneqq \mathcal{M}^{\prime}$. Let $j \in \mathcal{M}^{\prime} \backslash \mathcal{M}$ (hence $j \in \mathcal{H}$ ). Since player $j$ is one of the expensive players in equilibrium $\sigma$ and one of the medium players in equilibrium $\sigma^{\prime}$, and, as we have 
shown, $z_{q}=z_{q}^{\prime}$, it must be the case that $z_{j}>z_{j}^{\prime}$. We now show that this is not possible.

The following equation holds for a player in $\mathcal{M} \cup \mathcal{H}$ (and hence for player $j$, since $j \in \mathcal{H}$ ):

$$
z_{j}=\frac{\delta}{n}\left[1-Z_{q}+z_{q}\right]+\delta\left(\mu_{j}-\frac{1}{n}\right) z_{j}+(1-\delta) r_{j} .
$$

Collecting terms in $j$, we find

$$
\left(1+\frac{\delta}{n}-\delta \mu_{j}\right) z_{j}=\frac{\delta}{n}\left[1-Z_{q}+z_{q}\right]+(1-\delta) r_{j}
$$

An analogous equation holds for a player in $\mathcal{M}^{\prime} \cup \mathcal{H}^{\prime}$ (and hence for player $j$, since $j \in \mathcal{M}^{\prime}$ ):

$$
\left(1+\frac{\delta}{n}-\delta \mu_{j}^{\prime}\right) z_{j}^{\prime}=\frac{\delta}{n}\left[1-Z_{q}^{\prime}+z_{q}^{\prime}\right]+(1-\delta) r_{j} .
$$

By assumption, $Z_{q}^{\prime}=Z_{q}$ and, as we have shown, this implies $z_{q}^{\prime}=z_{q}$. Hence, player $j$ gets the same payoff as proposer in both equilibria. The only way in which $z_{j}>z_{j}^{\prime}$ is if $\mu_{j}>\mu_{j}^{\prime}$. However, since $j \in \mathcal{H}, j$ is never a responder in equilibrium $\sigma$ but may be a responder in equilibrium $\sigma^{\prime}$, implying $z_{j} \leq z_{j}^{\prime}$, a contradiction.

1. b) Suppose $Z_{q} \neq Z_{q}^{\prime}$. Without loss of generality, let $Z_{q}<Z_{q}^{\prime}$.

Coming back to equation (10), this implies $z_{i}>z_{i}^{\prime}$ for all $i \in \mathcal{L} \cap \mathcal{L}^{\prime}$. Given that $\mathcal{L}$ and $\mathcal{L}^{\prime}$ coincide, it follows that $Z_{L}>Z_{L^{\prime}}^{\prime}$, which together with $Z_{q}<Z_{q}^{\prime}$ implies $z_{q}<z_{q}^{\prime}$.

Consider the set $\mathcal{M} \cap \mathcal{M}^{\prime}$ (clearly, this set is nonempty since $q \in \mathcal{M} \cap$ $\mathcal{M}^{\prime}$ ). For any $i \in \mathcal{M}, z_{i}=z_{q}$, so (replacing $z_{i}=z_{q}$ in (12) and collecting terms) we can write $i$ 's equilibrium continuation value as

$$
\left(1-\delta \mu_{i}\right) z_{q}=\frac{\delta}{n}\left(1-Z_{q}\right)+(1-\delta) r_{i}
$$

Analogously, for any $i \in \mathcal{M}^{\prime}$,

$$
\left(1-\delta \mu_{i}^{\prime}\right) z_{q}^{\prime}=\frac{\delta}{n}\left(1-Z_{q}^{\prime}\right)+(1-\delta) r_{i}
$$

Given that $Z_{q}^{\prime}>Z_{q}$ but $z_{q}<z_{q}^{\prime}$, it must be the case that $\mu_{i}^{\prime}>\mu_{i}$.

Now consider the total probability of being involved in a coalition in equilibrium. The total probability is $\sum_{i \in N} \mu_{i}=\sum_{i \in N} \mu_{i}^{\prime}=q$. Further, each player must be included if he is selected to be the proposer, 
hence $\mu_{i} \geq \frac{1}{n}$ for all $i$. There is then a total probability of being coalition partner of $q-1$, which may be distributed differently in the two equilibria. Given that players in $\mathcal{H}$ (respectively $\mathcal{H}^{\prime}$ ) never get proposals, we have a total probability of $q-1$ to be distributed between players in $\mathcal{L} \cup \mathcal{M}$ in equilibrium $\sigma$, and between players in $\mathcal{L}^{\prime} \cup \mathcal{M}^{\prime}$ in equilibrium $\sigma^{\prime}$. Further, note that all players in $\mathcal{L}$ have $\mu_{i}=1$, and all players in $\mathcal{L}^{\prime}$ have $\mu_{i}^{\prime}=1$. Since by assumption $\mathcal{L}=\mathcal{L}^{\prime}$, we have $\sum_{i \in \mathcal{M}}\left(\mu_{i}-\frac{1}{n}\right)=\sum_{i \in \mathcal{M}^{\prime}}\left(\mu_{i}^{\prime}-\frac{1}{n}\right)$, and the only way in which $\mu_{i}^{\prime}>\mu_{i}$ for all players in $\mathcal{M} \cap \mathcal{M}^{\prime}$ is if $\mathcal{M}^{\prime} \varsubsetneqq \mathcal{M}$ (otherwise we would "run out of probability").

Let $j$ be a player in $\mathcal{M} \backslash \mathcal{M}^{\prime}$. If we consider equilibrium $\sigma$, using (11) we have (since the worst-case scenario is $\mu_{j}=\frac{1}{n}$ ):

$$
\begin{aligned}
z_{j} & =z_{q} \geq \frac{\delta}{n}\left(1-Z_{q}+z_{q}\right)+(1-\delta) r_{i} \\
\left(1-\frac{\delta}{n}\right) z_{q} & \geq \frac{\delta}{n}\left(1-Z_{q}\right)+(1-\delta) r_{i}
\end{aligned}
$$

If we consider equilibrium $\sigma^{\prime}$, where $j \in \mathcal{H}^{\prime}$, we have

$$
\begin{aligned}
z_{q}^{\prime} & <z_{j}^{\prime}=\frac{\delta}{n}\left(1-Z_{q}^{\prime}+z_{q}^{\prime}\right)+(1-\delta) r_{i} \\
\left(1-\frac{\delta}{n}\right) z_{q}^{\prime} & <\frac{\delta}{n}\left(1-Z_{q}^{\prime}\right)+(1-\delta) r_{i}
\end{aligned}
$$

Putting the two expressions together, since $Z_{q}^{\prime}>Z_{q}$, we find

$$
\left(1-\frac{\delta}{n}\right) z_{q}^{\prime}<\frac{\delta}{n}\left(1-Z_{q}^{\prime}\right)+(1-\delta) r_{i}<\frac{\delta}{n}\left(1-Z_{q}\right)+(1-\delta) r_{i} \leq\left(1-\frac{\delta}{n}\right) z_{q}
$$

which implies $z_{q}^{\prime}<z_{q}$, a contradiction.

Case 2. Suppose $\mathcal{L} \neq \mathcal{L}^{\prime}$. Without loss of generality, let $\mathcal{L} \varsubsetneqq \mathcal{L}^{\prime}$. There are two possible cases, depending on how $Z_{q}$ compares with $Z_{q}^{\prime}$.

2. a) Suppose $Z_{q} \leq Z_{q}^{\prime}$. We can write $Z_{q} \leq Z_{q}^{\prime}$ as

$$
\sum_{i \in \mathcal{L}} z_{i}+(q-L) z_{q} \leq \sum_{i \in \mathcal{L}} z_{i}^{\prime}+\sum_{i \in \mathcal{L}^{\prime} \backslash \mathcal{L}} z_{i}^{\prime}+\left(q-L^{\prime}\right) z_{q}^{\prime}
$$

By equation $(10), Z_{q} \leq Z_{q}^{\prime}$ implies $z_{i} \geq z_{i}^{\prime}$ for all $i \in \mathcal{L} \cap \mathcal{L}^{\prime}$, hence

$$
(q-L) z_{q} \leq \sum_{i \in \mathcal{L}^{\prime} \backslash \mathcal{L}} z_{i}^{\prime}+\left(q-L^{\prime}\right) z_{q}^{\prime}
$$


By definition, $z_{i}^{\prime}<z_{q}^{\prime}$ for all $i \in \mathcal{L}^{\prime}$. Hence, the equation above indicates that $z_{q}$ is at most as large as a weighted average of several values, the largest of which is $z_{q}^{\prime}$. Thus, $z_{q}<z_{q}^{\prime}$.

If we now look at players $j \in \mathcal{M} \cap \mathcal{M}^{\prime}$ (a set that includes player $q$ ), comparing equations (14) and (15) we see that, given that $Z_{q} \leq Z_{q}^{\prime}$, the only way in which $z_{q}<z_{q}^{\prime}$ is if $\mu_{i}<\mu_{i}^{\prime}$ for all these players.

Recall that the probability of being a coalition partner is $\mu_{i}-\frac{1}{n}\left(\mu_{i}^{\prime}-\frac{1}{n}\right.$ in equilibrium $\left.\sigma^{\prime}\right)$. It holds that $\sum_{i \in \mathcal{L} \cup \mathcal{M}}\left(\mu_{i}-\frac{1}{n}\right)=\sum_{i \in \mathcal{L}^{\prime} \cup \mathcal{M}^{\prime}}\left(\mu_{i}^{\prime}-\frac{1}{n}\right)^{n}=$ $q-1$. Consider the allocation of this probability, starting by player 1 onwards. Players in $\mathcal{L} \cap \mathcal{L}^{\prime}$ (i.e., players in $\mathcal{L}$ ) have $\mu_{i}=\mu_{i}^{\prime}=1$. Players in $\mathcal{M} \cap \mathcal{L}^{\prime}$ have $\mu_{i} \leq \mu_{i}^{\prime}=1$. Players in $\mathcal{M} \cap \mathcal{M}^{\prime}$ have $\mu_{i}<\mu_{i}^{\prime}$. It then follows that $\mathcal{L}^{\prime} \cup \mathcal{M}^{\prime} \subsetneq \mathcal{L} \cup \mathcal{M}$, that is, the total probability $q-1$ must be exhausted earlier in the equilibrium $\sigma^{\prime}$. Hence, the set $\mathcal{M} \backslash\left(\mathcal{L}^{\prime} \cap \mathcal{M}^{\prime}\right)$ is nonempty. Let $j$ be a player in this set. Player $j$ is in set $\mathcal{M}$ in the equilibrium $\sigma$, but is in set $\mathcal{H}^{\prime}$ in equilibrium $\sigma^{\prime}$. Since $z_{q}<z_{q}^{\prime}$, this implies $z_{j}=z_{q}<z_{q}^{\prime}<z_{j}^{\prime}$. We can then find a contradiction by the same reasoning as in case $1 b$ ).

2. b) Let $Z_{q}>Z_{q}^{\prime}$. Then $z_{i}<z_{i}^{\prime}$ for all $i \in \mathcal{L}$. We then have

$$
Z_{q}=\sum_{i \in \mathcal{L}} z_{i}+(q-L) z_{q}>\sum_{i \in \mathcal{L}} z_{i}^{\prime}+\sum_{i \in \mathcal{L}^{\prime} \backslash \mathcal{L}} z_{i}^{\prime}+\left(q-L^{\prime}\right) z_{q}^{\prime}=Z_{q}^{\prime} .
$$

Hence, $(q-L) z_{q}>\sum_{i \in \mathcal{L}^{\prime} \backslash \mathcal{L}} z_{i}^{\prime}+\left(q-L^{\prime}\right) z_{q}^{\prime}$. This does not give us a clear relationship between $z_{q}$ and $z_{q}^{\prime}$, though it tells us that $z_{q}>z_{i}^{\prime}$ for some $i \in \mathcal{L}^{\prime} \backslash \mathcal{L}$. In other words, there is a player in $\mathcal{M} \cap \mathcal{L}^{\prime}$ with $z_{i}>z_{i}^{\prime}$. Consider the equations for $z_{i}$ and $z_{i}^{\prime}, i \in \mathcal{M} \cap \mathcal{L}^{\prime}$. Since $i \in \mathcal{M}$, we have

$$
\left(1-\delta \mu_{i}\right) z_{i}=\frac{\delta}{n}\left(1-Z_{q}\right)+(1-\delta) r_{i}
$$

On the other hand, since $i \in \mathcal{L}^{\prime}$, we have

$$
(1-\delta) z_{i}^{\prime}=\frac{\delta}{n}\left(1-Z_{q}^{\prime}\right)+(1-\delta) r_{i}
$$

Since $\mu_{i} \leq 1$ and $Z_{q}>Z_{q}^{\prime}$, it follows that $z_{i}^{\prime}>z_{i}$, a contradiction.

Hence, SSPE payoffs are unique if the sufficient conditions for immediate agreement are satisfied; if not, there may be multiple equilibria. 


\section{A.4 Limit results as $\delta \rightarrow 1$}

Proposition 6. If $q<n$, there is a value $\bar{\delta}<1$ such that the unique no-delay SSPE of the game $G(n, q, \delta, r)$ has $N=\mathcal{M}$ for $\bar{\delta}<\delta<1$.

Proof. $\mathcal{L}$ and $\mathcal{H}$ must both be empty when $\delta$ is sufficiently close to 1 . This can be proved by contradiction.

Suppose $\mathcal{L} \neq \varnothing$ in equilibrium for $\delta \rightarrow 1$. If we look at equation (6) for $z_{i}$ in $\mathcal{L}$, we see that $\frac{1-Z_{q}}{1-\delta}$ becomes unbounded when $q<n$ as $\delta \rightarrow 1$, a contradiction. This is because in a no-delay equilibrium we have $\sum_{i \in N} z_{i}=\delta+(1-\delta) \sum_{i \in N} r_{i}$, therefore the largest continuation value must be at least $\frac{\delta}{n}+(1-\delta) \bar{r}_{N}$. Since $q<n$, $Z_{q} \leq \sum_{i \in N} z_{i}-z_{n} \leq \delta \frac{n-1}{n}+(n-1)(1-\delta) \bar{r}_{N}$, and $1-Z_{q} \geq 1-\delta \frac{n-1}{n}-(n-1)(1-\delta) \bar{r}_{N}$. When $\delta \rightarrow 1$, the lower bound for $1-Z_{q}$ approaches $\frac{1}{n}>0$. More generally, $\mu_{i}<1$ for all $i$ for $\delta$ sufficiently large (also for players in $\mathcal{M}$ ), as otherwise we could rewrite (3) and find that $z_{q}$ becomes unbounded in the same way.

Similarly, suppose $\mathcal{H} \neq \varnothing$ and take player $n \in \mathcal{H}$. Let $m$ be the player with the smallest $r_{i}$ in $\mathcal{M}$ (in general the identity of player $m$ may depend on $\delta$ ). We now show that $z_{m}-z_{n}=\delta\left(\mu_{m}-\frac{1}{n}\right) z_{q}-(1-\delta)\left(r_{n}-r_{m}\right)>0$ for $\delta$ close enough to 1 , a contradiction. To see this, note that expression $(1-\delta)\left(r_{n}-r_{m}\right)$ clearly converges to 0 , since $r_{n}-r_{m} \leq r_{n}-r_{1}$ and $\delta \rightarrow 1$. It remains to be shown that $\left(\mu_{m}-\frac{1}{n}\right) z_{q}$ remains strictly positive as $\delta \rightarrow 1$. First we show that $\mu_{m}>\frac{1}{n}$ for all values of $\delta$. Given that $\mathcal{L}=\varnothing$ for a sufficiently large value of $\delta$ and $q \geq 2$, $\sum_{i \in \mathcal{M}} \mu_{i}=q-\frac{H}{n}=q-1+\frac{M}{n} \geq 1+\frac{M}{n}$. Since $m$ is the player with the smallest value of $r_{i}$, he is also the player with the highest $\mu_{i}$ in $\mathcal{M}$ according to equation (3), hence $\mu_{m} \geq \frac{1}{M}+\frac{1}{n} \geq \frac{2}{n}$. It remains to be shown that $z_{q}>0$ for $\delta \rightarrow 1$. This is clear since, using $(3), z_{q} \geq \frac{\delta}{n}\left(1-Z_{q}\right)+(1-\delta) r_{m}$, and we have established that $1-Z_{q}$ remains positive as $\delta \rightarrow 1$. Hence the set $\mathcal{H}$ is empty for $\delta$ close enough to 1.

Corollary 4. In the limit when $\delta \rightarrow 1, y_{i}=\frac{1}{n}$ for all $i \in N{ }^{42}$

Hence, there is a sharp contrast between the results for $q=n$ and for $q<n$ when $\delta \rightarrow 1$. Under unanimity rule, the $r$-values remain relevant even if $\delta \rightarrow 1$. Under any majoritarian rule, continuation values are less sensitive to the $r$-values, which is consistent with players being unable to unilaterally secure these values, and these values become irrelevant in the limit as $\delta \rightarrow 1$.

\section{A.5 Equilibria with delay}

Consider a SSPE that involves delay, i.e. at least one player, when proposing, makes a proposal that is not accepted. Call him player $i$. Then it follows from

\footnotetext{
${ }^{42}$ When all players are in $\mathcal{M}$ we have $z_{q}=\frac{\delta}{n}+(1-\delta) \bar{r}_{N}$. Putting this together with the equation $z_{q}=\delta y_{i}+(1-\delta) r_{i}$, we see that both $z_{i}$ and $y_{i}$ converge to $\frac{1}{n}$ as $\delta \rightarrow 1$.
} 
Lemma 1 and our tie-breaking rule that the cheapest $q$-coalition including Mr. $i$ has $\sum_{S} z_{j}>1$. This in turn implies that no other player will build a coalition that includes Mr. $i$. Thus, the only way that Mr. $i$ influences the game is that he will 'stall' when it is his turn to propose, thereby increasing the chance that breakdown will occur before an 'active' player is chosen.

Suppose there are $k$ such 'stallers' in equilibrium. The remaining players make proposals that are accepted, and so they are essentially playing an equilibrium with immediate agreement, however in a 'transformed' game in which the probability of breakdown is increased. What is the new probability of breakdown?

After a proposal fails, breakdown will occur immediately with probability (1 $\delta)$. If not, then with probability $\frac{k}{n}$, a staller will propose next, in which case failure will again occur with probability $(1-\delta)$, etc. So the probability that breakdown will occur is

$$
(1-\tilde{\delta})=(1-\delta)+\frac{\delta k}{n}\left[(1-\delta)+\frac{\delta k}{n}(\cdots)\right]=1-\frac{\delta(n-k)}{n-\delta k}
$$

and so the modified continuation probability is

$$
\tilde{\delta}=\frac{\delta(n-k)}{n-\delta k}
$$

which is equal to $\delta$ when $k=0$ and equal to zero when $k=n$.

The expected payoffs and continuation values of the remaining $n-k$ players are determined as in equilibria with immediate agreement. For the 'stallers', expected payoffs are simply

$$
y_{j}=\rho r_{j}
$$

where $\rho$ is the probability that breakdown will occur before an active player is chosen to propose.

$$
\rho=\frac{k}{n}(1-\tilde{\delta})=\frac{(1-\delta) k}{n-\delta k}
$$

Two cases are possible: (1) If $k \leq n-q$, agreement will occur if one of the nonstallers is chosen to propose. The payoffs and continuation values of these players satisfy exactly the conditions we have previously derived. And the continuation values of the stallers must be strictly greater than the others. (2) $k=n$, i.e. no agreement occurs.

\section{A.6 Proof of propositions 1 and 2}

Propositions 1 and 2 easily follow from the results of previous sections. 
Proof of proposition 1. If $\sum_{i \in N} r_{i}<1$, all SSPE must have immediate agreement by corollary 1 . If $\sum_{i \in N} r_{i}>1$, each player can ensure disagreement by rejecting all proposals and proposing $x_{i}=1$, hence $\sum_{i \in N} z_{i}>1$ and no agreement can occur. If $\sum_{i \in N} r_{i}=1$, it is an equilibrium for all players to propose $x=r$ and accept any proposal with $x_{i} \geq r_{i}$, and this is the equilibrium selected by our tie-breaking rule.

In a no-delay equilibrium under unanimity rule, each player offers the other $n-1$ players their continuation value and $\mu_{i}=1$ for all players, hence

$$
y_{i}=\frac{1}{n}\left[1-\sum_{j \in N \backslash\{i\}} z_{j}\right]+\frac{n-1}{n} z_{i}=\frac{1}{n}\left[1-\sum_{j \in N} z_{j}\right]+z_{i}
$$

Continuation values are related to expected payoffs by the equation

$$
z_{j}=\delta y_{j}+(1-\delta) r_{j}
$$

If we add up equations (17) and take into account that $\sum_{j \in N} y_{j}=1$ in a no-delay equilibrium,

$$
\sum_{j \in N} z_{j}=\delta+(1-\delta) \sum_{j \in N} r_{j}
$$

If we take equation (16) and replace $z_{i}$ by its value from (17) and $\sum_{j \in N} z_{j}$ by its value from (18), we obtain an equation with $y_{i}$ as the only unknown. Solving this equation, we get $y_{i}=\frac{1}{n}\left[1-\sum_{j \in N} r_{j}\right]+r_{i}$, and, using (17), $z_{i}=\frac{\delta}{n}\left[1-\sum_{j \in N} r_{j}\right]+r_{i}$.

Proof of Proposition 2. Parts (i)-(iii) follow directly from corollary 1, proposition 5 and lemma 2 respectively.

(iv) In a no-delay equilibrium, players in $\mathcal{L}$ are included in the final coalition with probability 1 (the maximum possible), and players in $\mathcal{H}$ are included in the final coalition with probability $\frac{1}{n}$ (the minimum possible, since proposers always include themselves). Within the set $\mathcal{M}$, the probability of inclusion is decreasing in $r_{i}$ as can be seen from equation (3). The result also holds in an equilibrium with delay, since any "stallers" will have the highest disagreement values and will be excluded for sure.

(v) This is a consequence of proposition 4. Since expected equilibrium payoffs are increasing within $\mathcal{L}$, decreasing within $\mathcal{M}$ and constant (but not higher than those in $\mathcal{M}$ ) within $\mathcal{L}$, there are four possible cases:

If $\mathcal{N}=\mathcal{M}$, expected payoffs are decreasing in the $r$-values.

If only $\mathcal{M}$ and $\mathcal{H}$ are nonempty, expected payoffs are first decreasing and then constant in the $r$-values.

If only $\mathcal{L}$ and $\mathcal{M}$ are nonempty, expected payoffs are first increasing and then decreasing in the $r$-values.

If all three sets are nonempty, expected payoffs are first increasing, then decreasing and then constant in the $r$-values. 


\section{A.7 The three-player case}

The three-player case can be solved by enumeration. With three players there are only four possible types of no-delay equilibria: $L=1$ and $M=2 ; L=1, M=1$ and $H=1 ; M=3 ; M=2$ and $H=1$. For each of these four possible types we can find conditions on the parameters $r$ and $\delta$ in order for this type of equilibrium to exist. The no-delay equilibrium is unique as we know from proposition 5 , hence each parameter combination is compatible with only one type of equilibrium.

Proposition 7. Let $r_{1}+r_{3}<1$. There is a unique no-delay SSPE for each combination of $r$ and $\delta$. The no-delay SSPE is of one of four possible types:

a) $L=1, M=1, H=1$ occurs if $r_{1}<r_{2}<r_{3}$ and $\delta<\delta$.

b) $L=1, M=2$ occurs if $r_{2}>\frac{r_{1}+r_{3}}{2}$ and $\underline{\delta} \leq \delta<\bar{\delta}$.

c) $M=2, H=1$ occurs if $r_{2}<\frac{r_{1}+r_{3}}{2}$ and $\underline{\delta} \leq \delta<\bar{\delta}$.

d) $M=3$ occurs if $\delta \geq \bar{\delta}$.

The idea of the proof is as follows. We first conjecture a particular value for $L$, $M$ and $H$ (for example, $L=1$ and $M=2$ ). This conjecture leads to a system of equations that can be solved for $z_{i}$ and $\mu_{i}$ (see section A.3). In order for the solution to be an equilibrium, the found values of $z_{i}$ must be consistent with our initial conjecture (in the example, the found value of $z_{1}$ must be below the found value of $z_{2}=z_{3}$ ), and any mixed strategies that are played must involve probabilities between 0 and 1 (in the example, player 1 is mixing between proposing to player 2 and proposing to player 3). These conditions leads to inequalities involving the parameters $r_{1}, r_{2}, r_{3}$ and $\delta$.

Even though the proof is quite lengthy, the intuition behind the result is clear.

First, there are some conditions on the $r_{i}$ values. In particular, case $L=1$, $M=1, H=1$ is only possible if all values are different. $L=1, M=2$ is only possible if $r_{2}>\frac{r_{1}+r_{3}}{2}$, so that $r_{2}$ and $r_{3}$ are close together relative to $r_{1}$ and players 2 and 3 can be grouped together in the same class. $M=2, H=1$ is only possible if $r_{2}<\frac{r_{1}+r_{3}}{2}$ so that players 1 and 2 can be grouped together in the same class.

Second, there are some conditions on $\delta . L=1, M=1, H=1$ occurs when $r_{1}<r_{2}<r_{3}$ and $\delta=0$ since $z_{i}=r_{i}$ in this case. The inequality $z_{1}<z_{2}<z_{3}$ can be sustained as long as $\delta$ is sufficiently low, so that the difference in $r_{i}$ overrules the fact that players with a lower $r_{i}$ get more proposals. $L=1, M=2$ occurs when $z_{1}<z_{2}=z_{3}$, which can be sustained for $r_{2}>\frac{r_{1}+r_{3}}{2}$ given an intermediate value of $\delta$. On the one hand, $\delta$ needs to be sufficiently high so that player 1's strategy can compensate the difference between $r_{2}$ and $r_{3}$ by proposing to player 2 more often (if $r_{2}=r_{3}$ there is no difference to compensate, so $\underline{\delta}=0$ ), but sufficiently low to keep $z_{1}$ below $z_{2}$ despite player 1 getting more proposals. Likewise, $M=2$, $H=1$ occurs when $z_{1}=z_{2}<z_{3}$, which can be sustained for $r_{2}<\frac{r_{1}+r_{3}}{2}$ given an intermediate value of $\delta$. The continuation probability $\delta$ needs to be sufficiently 
high so that player 3's strategy can compensate the difference between $r_{1}$ and $r_{2}$, but sufficiently low to keep $z_{1}$ and $z_{2}$ below $z_{3}$, despite player 3 getting no proposals (if $r_{1}=r_{2}, \underline{\delta}=0$ ). Finally, $M=3$ needs a sufficiently high value of $\delta$ so that $z_{1}=z_{2}=z_{3}$ despite the possible differences between $r_{1}, r_{2}$ and $r_{3}$. The thresholds $\underline{\delta}$ and $\bar{\delta}$ have a different expression in terms of the $r$-values depending on whether $r_{2}<\frac{r_{1}+r_{3}}{2}$ or $r_{2}>\frac{r_{1}+r_{3}}{2}$ (both formulas are equivalent when $r_{2}=\frac{r_{1}+r_{3}}{2}$ ). The reason for this is that different inequalities are binding depending on the parameters. 